\title{
Gletscherarchäologie in den Österreichischen Alpen (GAAA)
}

\author{
Endbericht zu dem von der \\ Österreichischen Akademie der Wissenschaften \\ geförderten Projekt
}

Thomas Bachnetzer, Stephanie E. Metz, Beatrix Nutz und Harald Stadler
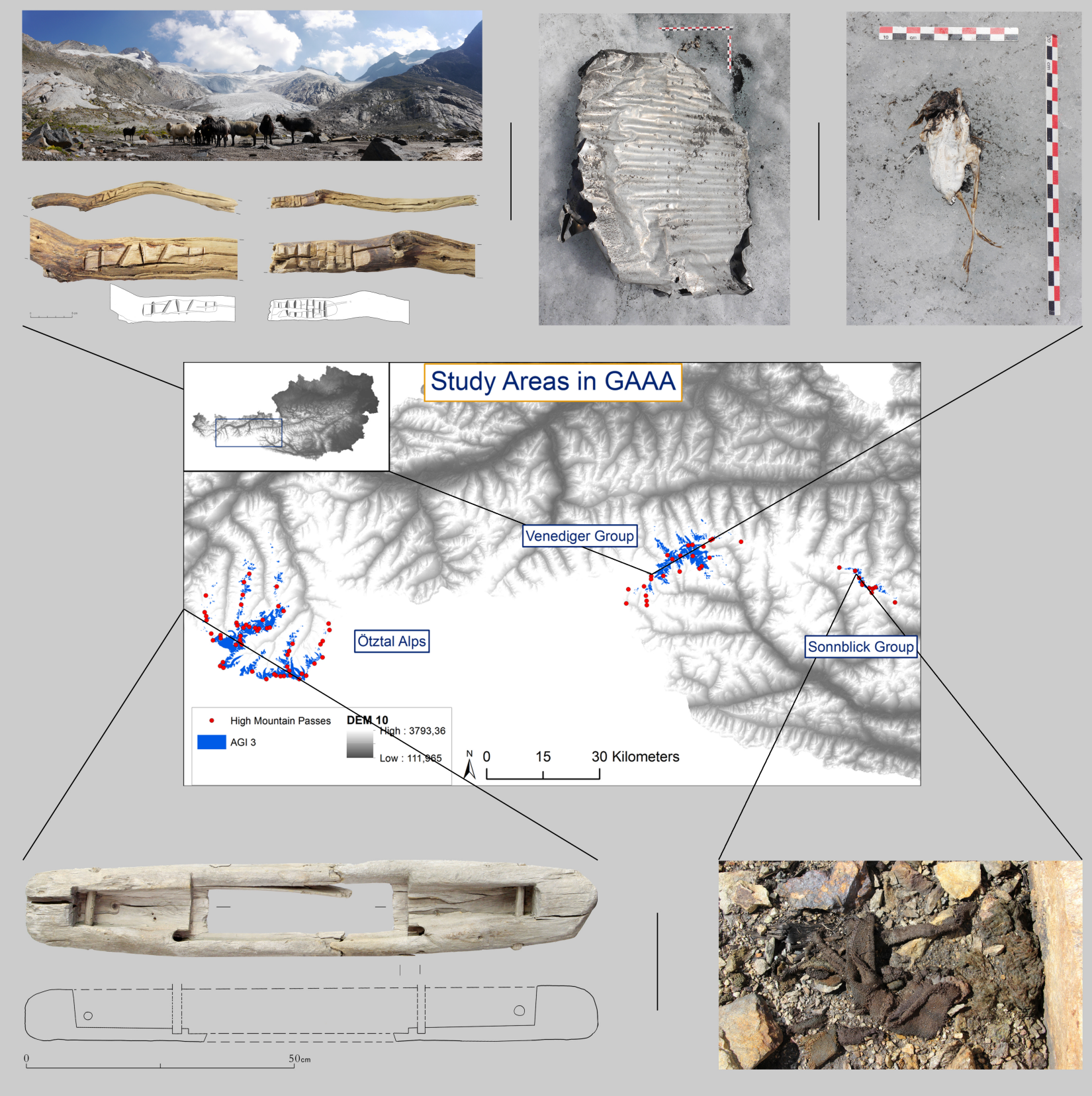

30 Kilometers
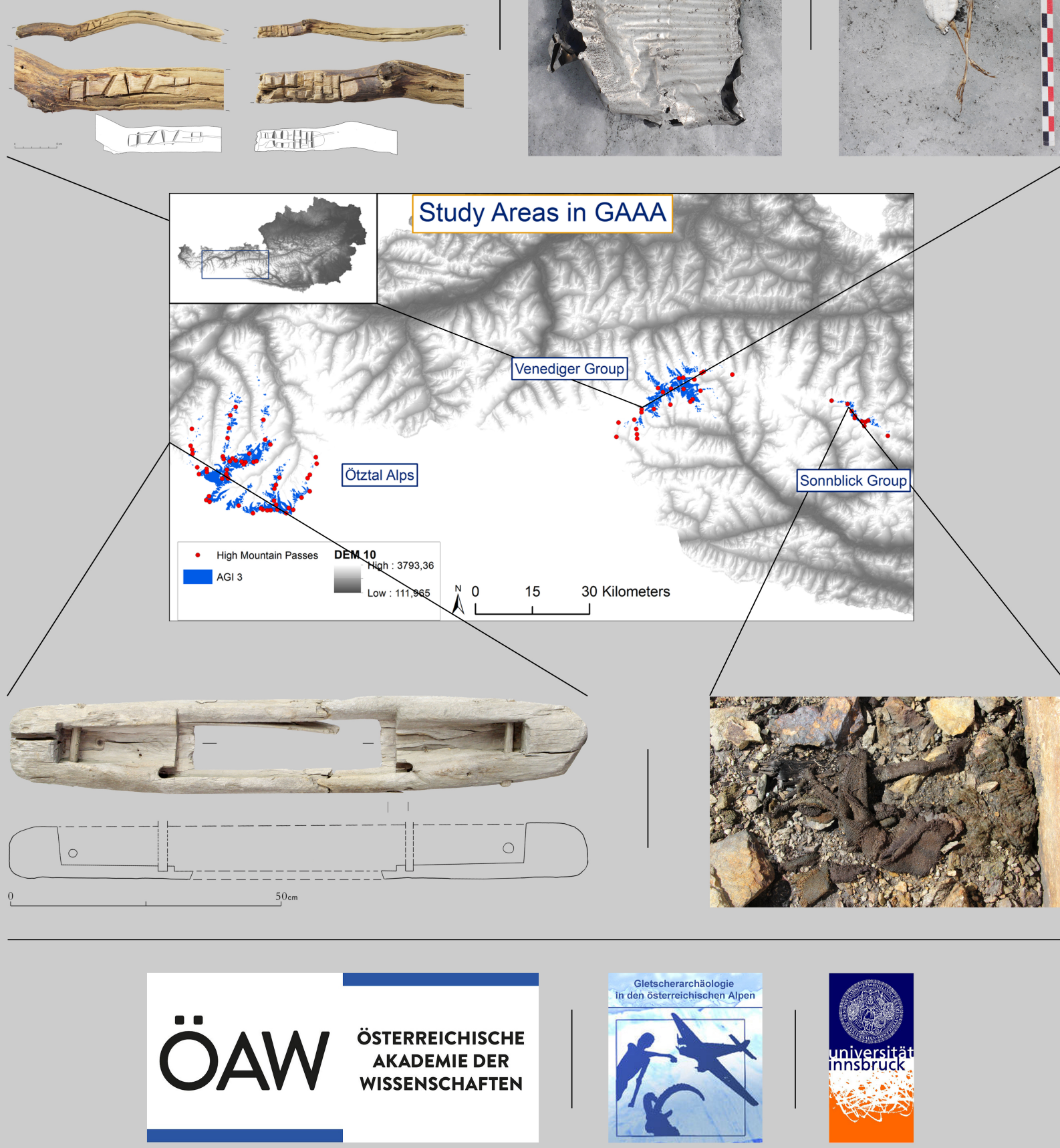


\section{Inhaltsverzeichnis}

1 Kurzfassung

2 Gletscherarchäologische Prospektionen und Vermessungen sowie gemeldete Gletscherfunde.... 3

2.1. Umbaltal / Umbalkees, Prägraten, Venediger Gruppe, Osttirol ................................................ 3

2.2. Vorderes Umbaltörl, Prägraten-Osttirol / Prettau-Südtirol, Venediger Gruppe, Osttirol............ 4

2.3. Goldzeche, Heiligenblut, Sonnblick Gruppe, Kärnten .......................................................... 6

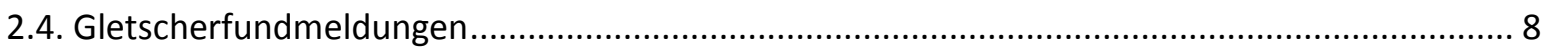

3 Predictive Modelling - Vorhersagemodell für gletscherarchäologische Funderwartungszonen... 11

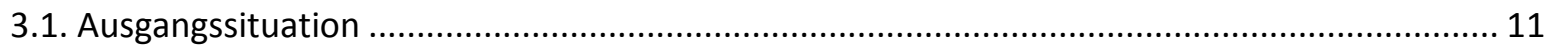

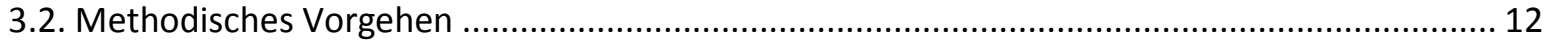

3.3. Fallstudie Venediger Gruppe - Umbaltal, Osttirol ................................................................... 17

4 Öffentlichkeitsarbeit im Rahmen von Zeitungsartikeln, Flyern, Foldern und Homepage ............. 19

5 Tagung , Workshop und Ausstellung / Prospektionen in angrenzenden Gebieten ....................... 20

5.1. Gletscherarchäologie-Tagung Frozen Pasts 4 (Innsbruck, 12.-16.10.2016) ............................. 20

5.2. Gletscherarchäologie-Workshop (Innsbruck, 22.12.2015) ........................................................ 21

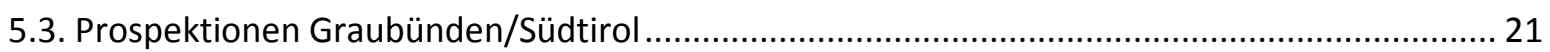

6 Vortragstätigkeit und Posterpräsentationen bei Tagungen ................................................... 22

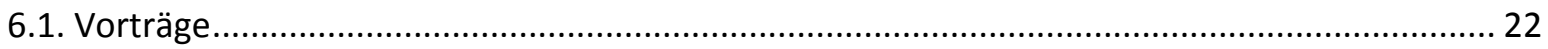

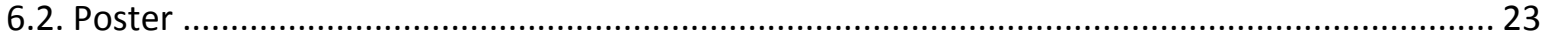

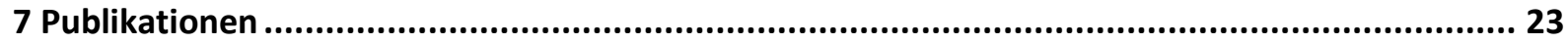

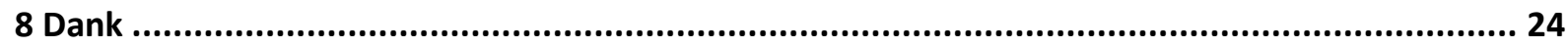

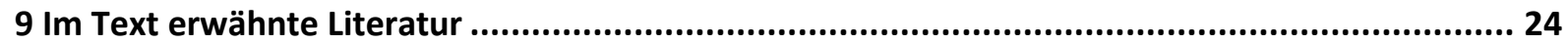

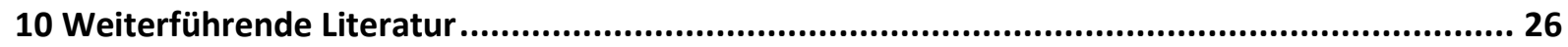




\section{Kurzfassung}

Das GAAA-Projekt (Glacial Archaeology in the Austrian Alps) baut auf einem 3-Säulensystem auf, das sich in Geländebegehungen, einem auf GIS basierenden Vorhersagemodell (Predictive Modelling) und Öffentlichkeitsarbeit unterteilen lässt. Im Rahmen von 20 Prospektionstagen konnten zahlreiche Artefakte geborgen und dokumentiert werden. Unter ihnen befindet sich ein am Vorderen Umbaltörl in Osttirol aus dem Eis ausgeapertes, mit Kerben versehenes Astfragment, das in den Zeitraum von $724-400$ v. Chr. datiert.

Im Rahmen des Projektes sollte ein GIS-basiertes Vorhersagemodell für gletscherarchäologische Funderwartungszonen erstellt werden. Dieses soll als Basis für gezielte Begehungen dienen, um eine maximale Zeitersparnis im Gelände zu ermöglichen. Unter Einbeziehung historischer und rezenter Gletscherstände werden Aussagen über zukünftige Gletscher- bzw. Eisflächenrückgänge getroffen, die in Korrelation mit bisherigen Fundaufkommen gesetzt werden. Basierend auf den physischen Charakteristika des Terrains können hierdurch Regionen definiert werden, die ein besonders hohes archäologisches Potenzial bieten bzw. besonders gefährdet sind. Das Modell ist noch in Ausarbeitung. Deshalb erfolgt die Berichterstattung mit den vorläufigen Ergebnissen.

Im Rahmen der Öffentlichkeitsarbeit erschienen 17 Beiträge und Artikel in Zeitungen und Magazinen, die einem breiten Publikum die Thematik Gletscherarchäologie auf eine leicht verständliche Art und Weise näherbringen sollen. Des Weiteren wurde die Verteilung der Gletscherarchäologie-Infoflyer auf gletschernahen Hütten vorangetrieben. Während der Ausführung des Projektes wurden vier Gletscherfunde von Berggehern abgegeben. Darunter befindet sich ein $107 \mathrm{~cm}$ langes bearbeitetes Holzartefakt noch unbekannter Funktion vom Seekarjoch in Pfunds, das laut ${ }^{14} \mathrm{C}$-Datierung in das Hochmittelalter zwischen 1223 und 1275 n. Chr. datiert. Mit insgesamt zehn Vorträgen und drei Postern wurden die Ergebnisse des GAAA-Projektes einem Fachpublikum im In- und Ausland präsentiert. Im Rahmen des GAAA-Projektes fanden 2016 in Innsbruck der vom ÖAW mitfinanzierte internationale Gletscherarchäologiekongress Frozen Pasts 4 mit Wissenschaftler und Wissenschaftlerinnen aus 11 Nationen und eine Gletscherarchäologieausstellung sowie 2015 ein Gletscherarchäologie-Workshop mit Fachleuten aus Tirol (Österreich), Graubünden (Schweiz) und Südtirol (Italien) statt.

Durch das GAAA-Projekt gelang es die Gletscherarchäologie als archäologische Disziplin in der öffentlichen Wahrnehmung zu etablieren und die wissenschaftlichen Grundlagen für zukünftige weiterführende Forschungen zu schaffen. 


\section{Gletscherarchäologische Prospektionen und Vermessungen sowie}

\section{gemeldete Gletscherfunde}

Aufgrund der späten Schneefälle in den Frühjahren 2015 und 2016 sowie den darauf folgenden früh einsetzenden Schneefällen im Herbst 2015 und 2016 waren die Schneestände auf den Gletschern dementsprechend sehr hoch. Trotz der geringen Zeitfenster war es während der Projektlaufzeit möglich an insgesamt 20 Prospektionstagen eine Reihe an prähistorischen, mittelalterlichen und neuzeitlichen Funden zu bergen.

\subsection{Umbaltal / Umbalkees, Prägraten, Venediger Gruppe, Osttirol}

Die hochalpinen Übergänge Vorderes und Hinteres Umbaltörl mit 2.926 m und 2.849 m Höhe im hinteren Umbaltal, Venediger Gruppe, verbinden das Virgental in Osttirol mit dem Windtal, einem Seitental des Tauferer Ahrntals, in Südtirol ${ }^{1}$. Die Korridorzugänge von der Osttiroler Seite her wurden in die Untersuchungen miteingebunden, um Hinweise auf eine mögliche Begehung in der Ur- und Frühgeschichte oder dem Mittelalter bereits im Talbereich nachweisen zu können ${ }^{2}$. Insgesamt konnten 35 Objekte, darunter 31 Abrisituationen, zwei Mauerstrukturen, eine Steinanhäufung sowie eine Felsinschrift (1941. JL Schlaiten) dokumentiert werden. An Funden sind im Talbereich 32 Schuhnägel, zwei Schuhbeschläge, sechs Münzen aus der Zwischenkriegszeit von 1924 und eine Sense mit Holzstiel zu nennen. Während die Münzen, die Schuhbeschläge und die Sense ausnahmslos in die Neuzeit zu datieren sind, ist die Altersbestimmung bei einigen Schuhnägeln noch nicht restlos geklärt. Vor allem Schuhnägel mit rundem Kopf können bis in die römische Zeitperiode zurückreichen, wie z. B. Funde vom Schnidejoch im Kanton Bern (Schweiz) belegen.
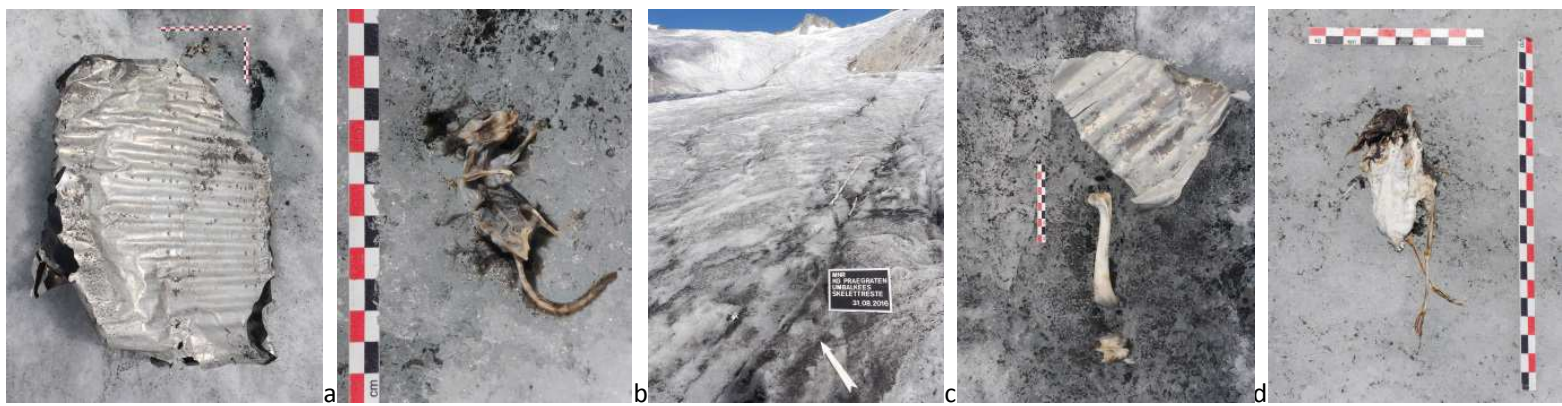

Abb. 1: Umbalkees, Prägraten, Osttirol. Aluteile Transportflugzeug JU52, Siebenschläfer, Rehbockskelettreste und mumifizierte Feldlerche. Foto: T. Bachnetzer, Inst. f. Archäologien, Univ. Innsbruck.

Im vorderen Bereich des Umbalkees konnten 211 Teile eines skelettierten Rehbocks (Abb. 1, c-d), eine mumifizierte Feldlerche (Abb. 1, e) und ein Siebenschläfer (Abb. 1, a-b) entdeckt werden, die

\footnotetext{
${ }^{1}$ Bodner 2003.

${ }^{2}$ Bachnetzer et al. 2017.
} 
allesamt laut ${ }^{14} \mathrm{C}$-Datierungen in die Neuzeit weisen. Des Weiteren wurden weitere Metallteile des 1941 am Umbalkees notgelandeten Transportflugzeuges Ju-52 geborgen (Abb. 1, a, d).

\subsection{Vorderes Umbaltörl, Prägraten-Osttirol / Prettau-Südtirol, Venediger Gruppe, Osttirol}

Die beiden Hauptziele der Prospektionen stellten das Vordere und Hintere Umbaltörl dar (Abb. 2). Während das Vordere Umbaltörl seit geraumer Zeit eisfrei ist, befinden sich auf dem Hinteren Umbaltörl noch kleinere Eisflächen, die jedoch im Sommer 2015 und 2016 mit Restschnee überlagert waren, sodass lediglich wenige schneefreie Bereiche abgesucht werden konnten.
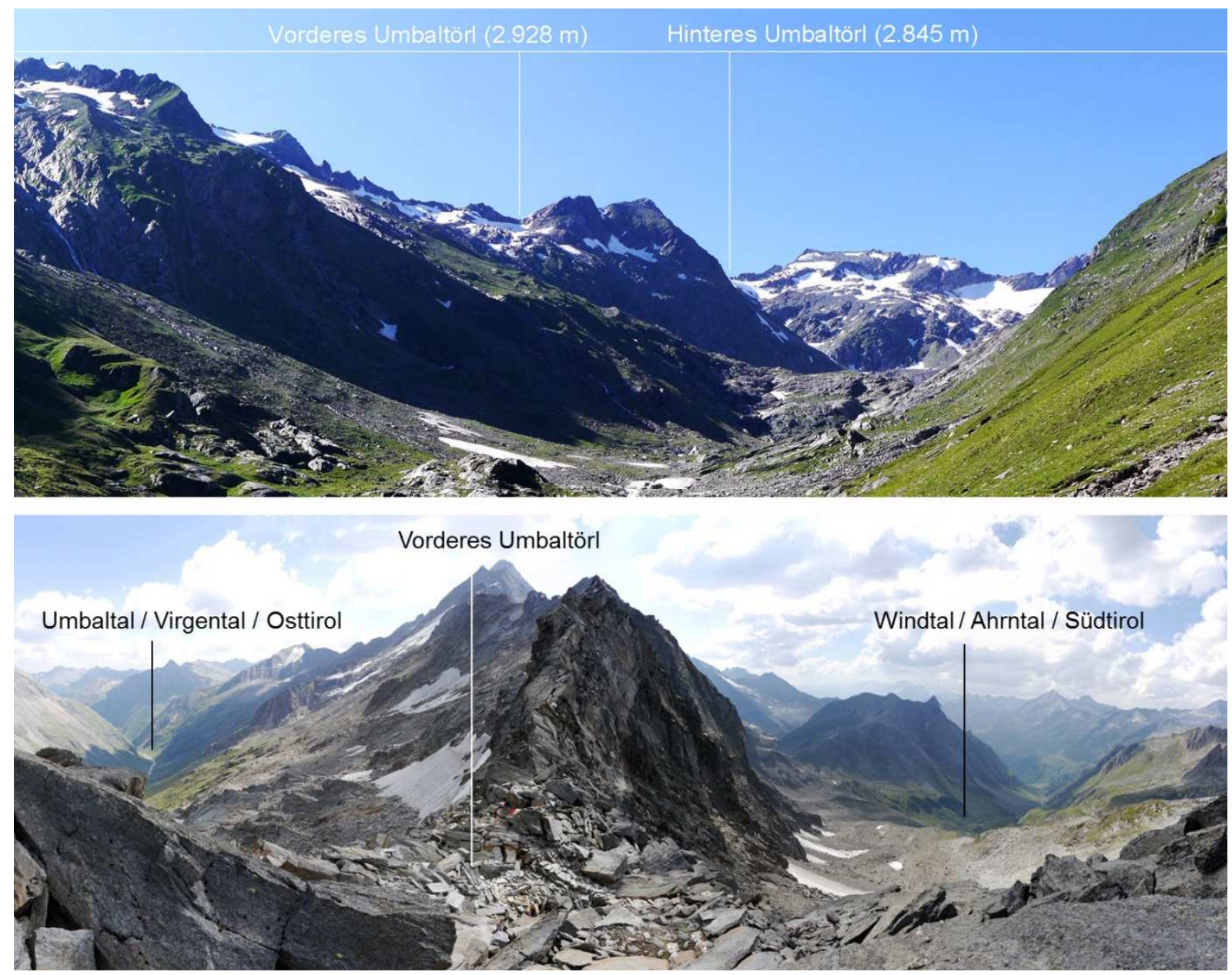

Abb. 2: Vorderes Umbaltörl, Prägraten-Osttirol / Prettau-Südtirol. Das Vordere Umbaltörl auf 2.928 m Höhe verbindet das Osttiroler Umbaltal mit dem Südtiroler Tauferer Ahrntal und ist seit geraumer Zeit gletscherfrei. Foto: T. Bachnetzer, Inst. f. Archäologien, Univ. Innsbruck.

Die Prospektionen konzentrierten sich dadurch auf das Vordere Umbaltörl, das von einer großen Blockschutthalde mit letzten Permafrostarealen zwischen den Steinblöcken geprägt ist. Die Begehungen lieferten insgesamt drei Nägel und 22 teils bearbeitete und verkohlte Hölzer (Abb. 4). Darunter befindet sich ein $24 \mathrm{~cm}$ langes leicht gekrümmtes Astfragment, das auf einer Seite neun längliche parallel geschnitzte Einkerbungen I|||||||| und auf einer weiteren Seite zwei fragmentierte V- 
förmige sowie zwei parallele Kerben aufweist VVII (Abb. 3) $)^{3}$. Das Stück ist auf einer Seite abgebrochen und auf der anderen Seite stark verwittert, sodass davon ausgegangen werden kann, dass die Einkerbungen in beide Richtungen eine Fortsetzung fanden. Laut ${ }^{14} \mathrm{C}$-Analyse weist das Artefakt in die Hallstattzeit (MAMS-25592: 2402+/-25 BP, cal BC 724-400, 95,4 \% Wahrscheinlichkeit). Damit zählt das Vordere Umbaltörl zu den wenigen bislang bekannten prähistorischen Gletscherfundplätzen in den Alpen ${ }^{4}$.
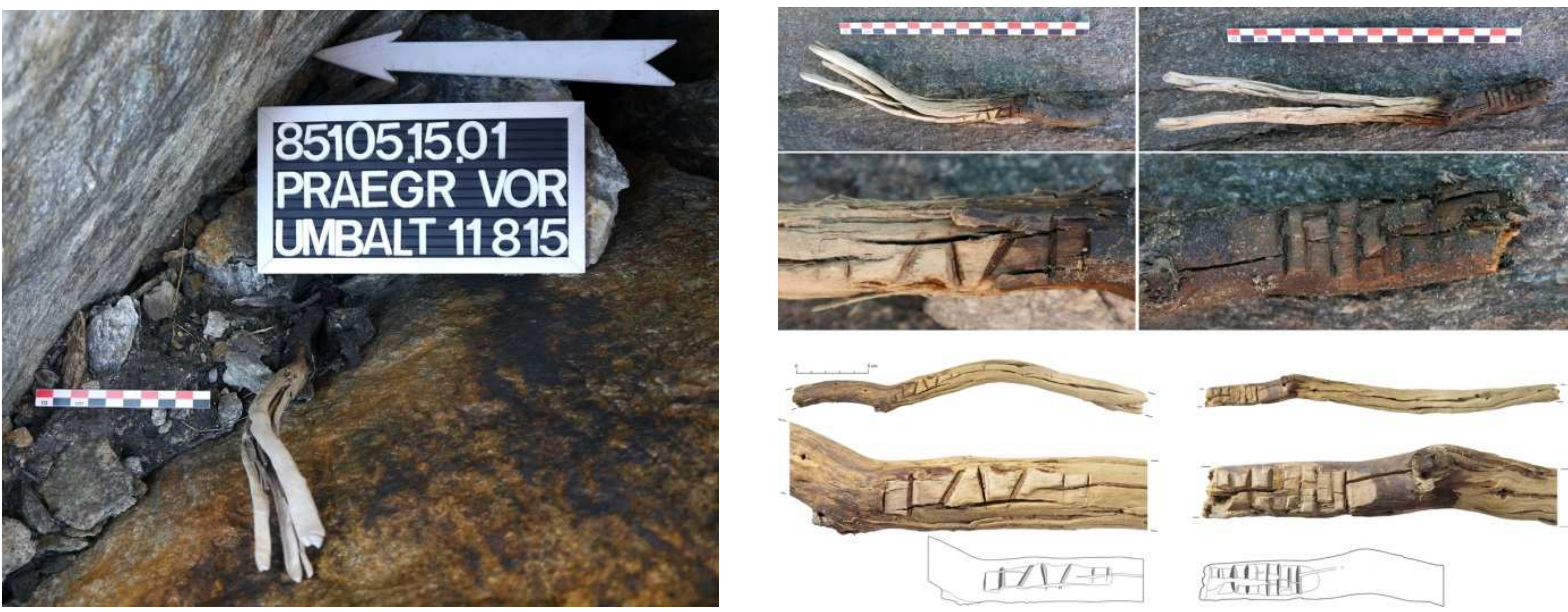

Abb. 3: Vorderes Umbaltörl, Prägraten-Osttirol / Prettau-Südtirol. Das gekerbte eisenzeitliche Astfragment in situ bzw. nach der Bergung und Restaurierung. Foto, Zeichnung: T. Bachnetzer, A. Blaickner, Inst. f. Archäologien, Univ. Innsbruck.
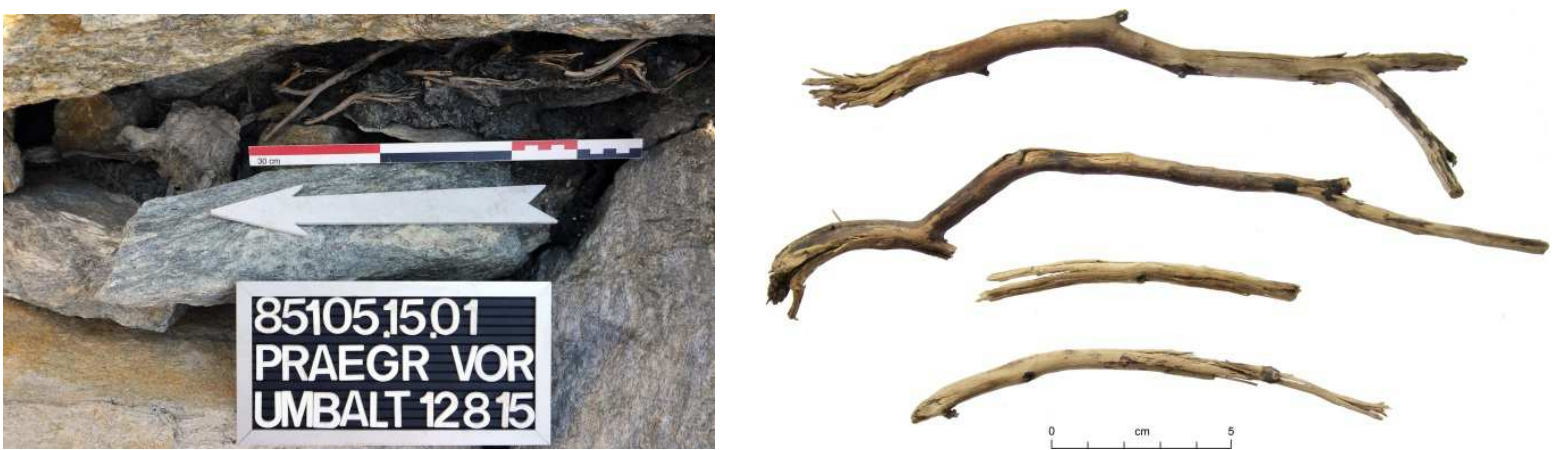

Abb. 4: Vorderes Umbaltörl, Prägraten-Osttirol / Prettau-Südtirol. Vier Astfragmente in situ und nach der Restaurierung. Foto, Zeichnung: T. Bachnetzer, Inst. f. Archäologien, Univ. Innsbruck.

Zur Bedeutung der Kerben gibt es nur wenige Anhaltspunkte. Laut Sprachwissenschaftlern handelt es sich nicht um Schriftzeichen im Sinne von Zeichen, die Sprache kodieren. In der Regel werden Formen wie I, V oder auch X gemeinhin als Marken bezeichnet, die in diesem Fall möglicherweise mit einer Art Zahlschreibung in Zusammenhang zu bringen sind. Bei einem der wenigen sehr guten

\footnotetext{
${ }^{3}$ Freundliche Mitteilung von Corinna Salomon, Thesaurus Inscriptionum Raeticarum, Institut für Sprachwissenschaften, Universität Wien.

${ }^{4}$ Die genaue topografische Verortung der Fundstücke vom Vorderen Umbaltörl ist noch nicht erfolgt. Da die Objekte im unmittelbaren Grenzbereich von Österreich und Italien entdeckt wurden, könnten sie sowohl auf Osttiroler als auch auf Südtiroler Seite gelegen haben.
} 
Vergleiche auf einem Holzartefakt handelt es sich um die 1,02 m lange Birkenrute vom Piperbühel bei Klobenstein am Ritten, Südtirol, Italien, die rätische Inschriften auf einer Länge von $22 \mathrm{~cm}$ aufweist ${ }^{5}$. Für eine dendrochronologische Analyse war das Objekt nicht geeignet zumal auch entsprechende Referenzreihen für Laubholzarten fehlen. Die Analyse beschränkte sich somit auf die Zählung der Jahrringe und die Bestimmung der Holzart unter einem möglichst geringen invasiven Eingriff. Am Querschnitt (Bruch) konnten in etwa 25 Jahrringe gezählt werden. Wie bei dieser Art von Proben üblich (Ast) nimmt der jährliche Zuwachs mit dem Alter stark ab, sodass im äußeren Bereich mit einer gewissen Unschärfe zu rechnen ist bzw. für eine genaue Auswertung ein entsprechender Querschnitt präpariert werden müsste. Am Querschnitt konnten im Zentrum sogenannte Markzellen beobachtet werden, damit ist eine Verwechslung mit einem Wurzelstück ausgeschlossen (Wurzeln bilden keine Markzellen aus). Für die Holzartbestimmung (SALIX) mussten Dünnschnitte (Tangential- und Radialschnitt) generiert werden, die unter dem Durchlichtmikroskop betrachtet werden konnten, damit bestimmte mikroskopische Merkmale die für eine Holzart sprechen identifiziert werden konnten (z.B. Breite der Markstrahlen, Durchbrechungen). Mit Hilfe der Probe aus dem T-Schnitt wurden die Markstrahlbreite erfasst: 1-reihig; im R-Schnitt der Typ der Markstrahlen, hier: heterogen, d.h. innen flachliegende Rechtecke, außen stehende Rechtecke; in den Kreuzungsfeldern treten auffallend große Tüpfel auf; die Durchbrechungen sind einfach: Im Gegenzug dazu gibt es die ev. leichter zu erkennenden leiterförmigen Durchbrechungen wie sie z.B. die Erle aufweist ${ }^{6}$.

Alle Holzartefakte wurden vom zuständigen Restaurator am Institut für Archäologien der Universität Innsbruck konserviert. Vor allem am Hinteren Umbaltörl kann bei anhaltendem Abschmelzen des Eises mit weiteren Funden gerechnet werden.

\subsection{Goldzeche, Heiligenblut, Sonnblick Gruppe, Kärnten}

In hochalpinen Bergbauregionen über 2500 m Seehöhe in den Hohen Tauern blieben Objekte aus organische Materialien wie Holz, Leder und Textil, die der Ausrüstung von Bergleuten zuzuordnen sind, unter Eis- und Schneefeldern sowie im gefrorenen Boden über die Jahrhunderte erhalten (Abb. 6-8) ${ }^{7}$. Unter den Textilien finden sich auch viele Reste von Bekleidung. Begehungen fanden an den ehemaligen Goldabbaustätten "Hinteres Freudental" im Großen Zirknitztal und "Goldzeche" am Zirmsee im Kleinfleiß Tal (Kärnten) statt. An der "Goldzeche" gelangten sieben Gebäudereste zur Vermessung (Abb. 5). Die Textil- und Lederfragmente können ins 15. bis 17. Jahrhundert datiert werden. Diese Datierung basiert einerseits auf der Betriebszeit der Bergwerke und dem Beginn der

\footnotetext{
${ }^{5}$ Mayr 1958. - Battisti 1934. - Ghislanzoni 1928.

${ }^{6}$ Die Holzartbestimmung wurde von Thomas Pichler am Institut für Geographie der Universität Innsbruck, Abteilung Dendrochronologie, durchgeführt.

${ }^{7}$ Nutz 2015. - 2016.
} 
so genannten Kleinen Eiszeit, eine Periode relativ kühlen Klimas die im 15. Jahrhundert beginnt und bis zum 19. Jahrhundert anhält. Ihr Höhepunkt in der Mitte des 17. Jahrhunderts und die Gletschervorstöße Mitte des 19. Jh. erschwerte den Bergbau in großen Höhen, wenn sie inn nicht gar unmöglich machte. Dies und die Erschöpfung der Goldadern setzten dem Bergbau im Laufe des 19. Jh. endgültig ein Ende. Andererseits konnten etliche Textilfragmente und Schuhe basierend auf ihrem Zuschnitt Modeerscheinungen zugeordnet werden. So bieten diese Funde eine sehr seltene, wenn nicht einzigartige Möglichkeit, Ausrüstung und Kleidung der frühneuzeitlichen Bergleute im Original zu studieren.
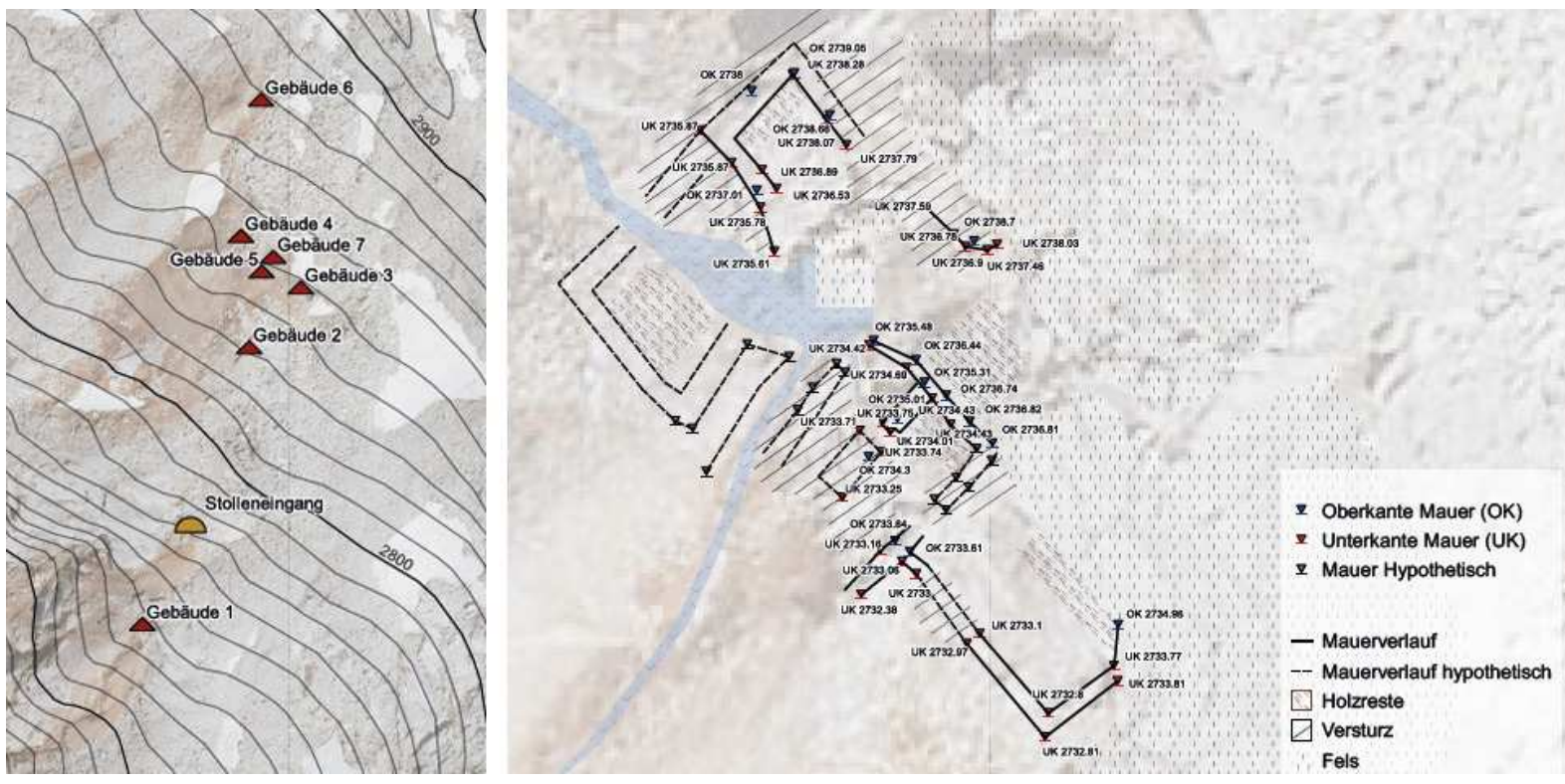

Abb. 5: Goldzeche, Heiligenblut, Kärnten. Plan der aufgenommenen Gebäudestrukturen und Gebäudestruktur 1. Plan: Fa. Ardis, Innsbruck.

\begin{tabular}{|c|c|c|c|c|c|c|c|}
\hline $\begin{array}{l}\text { Parzellen } \\
\text {-nummer }\end{array}$ & Datum & $\begin{array}{l}\text { Inventar } \\
\text {-nummer }\end{array}$ & Material & Objektbeschreibung & Größe in cm & \begin{tabular}{c|} 
Faden \\
-drehung \\
Kette
\end{tabular} & Bindung \\
\hline $945 / 2$ & 14.09.2016 & GZ 0167 & Glas & $\begin{array}{l}\text { Fuß und Kelchansatz eines "Fußkelchs, } \\
\text { Fußbecher". } \\
\text { Frühe Neuzeit } 1550 \text { bis etwa } 1650\end{array}$ & Dm. $4 \mathrm{~cm}$ & & \\
\hline $945 / 2$ & 14.09.2016 & GZ 0168 & Holz & Holzblatt eines Spatens? & $17 \times 10$ & & \\
\hline $945 / 2$ & 14.09.2016 & GZ 0169 & Holz & Daube & $18,3 \times 4,6$ & & \\
\hline $945 / 2$ & 14.09.2016 & GZ 0170 & Glas & Butzenscheiben-Fragment & $3 \mathrm{~cm}$ & & \\
\hline $945 / 2$ & 14.09.2016 & \begin{tabular}{l|} 
GZ $0171-$ \\
GZ 0175
\end{tabular} & Wolle & 5 Filzfragmente, eines mit Schlitzen & & & \\
\hline $945 / 2$ & 14.09.2016 & GZ 0176 & Leder & 3 Lederstücke, Schuhfragmente & & & \\
\hline $945 / 2$ & 14.09.2016 & GZ 0184 & Wolle & braun, dicht gewebt, mit Webkante & $40 \times 25$ & Z-Garn & Leinenbindung \\
\hline $945 / 2$ & 14.09.2016 & GZ 0185 & Wolle & $\begin{array}{c}\text { helleres braun, dicht gewebt, teils } \\
\text { verfilzt. Starke Fadendrehung - bis zu } \\
57^{\circ}\end{array}$ & $35 \times 19$ & Z-Garn & Leinenbindung \\
\hline $945 / 2$ & 14.09.2016 & GZ 0186 & Wolle & $\begin{array}{c}\text { grobes Wollfragment, Schussfäden (S- } \\
\text { Zwirn) in zwei Brauntönen }\end{array}$ & & Z-Garn & Spitzköperbindung \\
\hline $945 / 2$ & 14.09.2016 & GZ 0187 & Wolle & $\begin{array}{c}\text { dunkelbraun-schwarzer, grober } \\
\text { Wollstoff }\end{array}$ & $31 \times 15$ & S-Garn & Leinenbindung \\
\hline $945 / 2$ & 14.09.2016 & GZ 0188 & Wolle & $\begin{array}{l}\text { Fragment eines schwarzen Wollstoffes } \\
\text { in Leinenbindung }\end{array}$ & $23 \times 10$ & Z-Garn & Leinenbindung \\
\hline
\end{tabular}

Abb. 6: Goldzeche, Heiligenblut, Kärnten. Funde während der Vermessungsarbeiten. Fundaufnahme: B. Nutz, Inst. f. Archäologien, Univ. Innsbruck. 

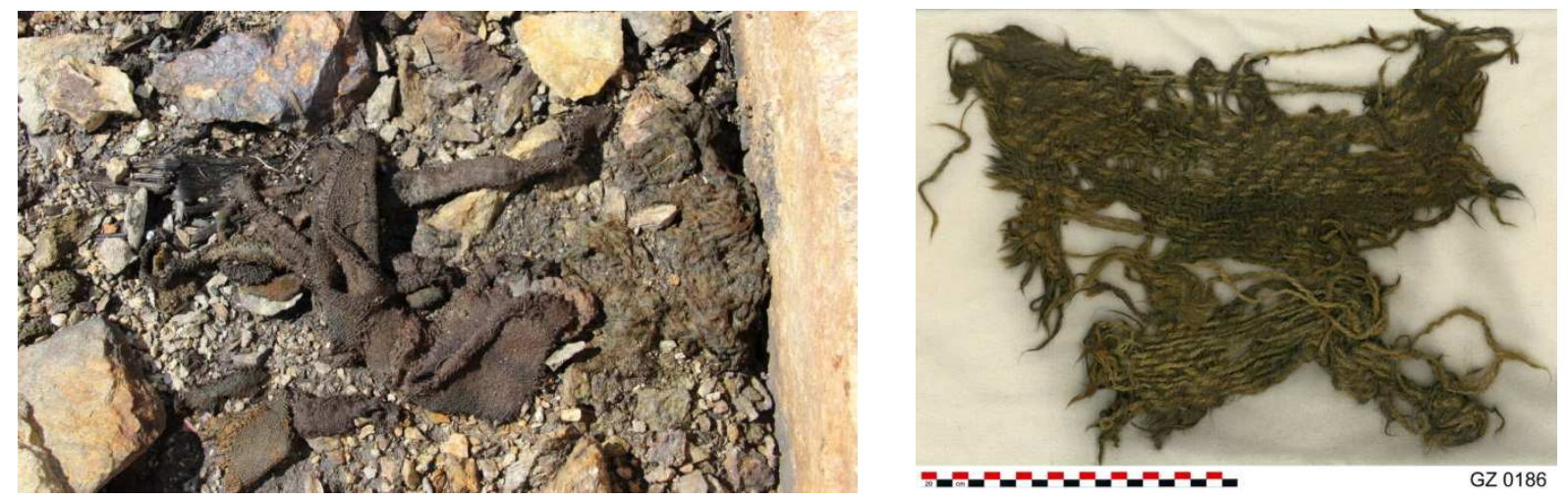

Abb. 7: Goldzeche, Heiligenblut, Kärnten. Textilreste bei der Auffindung innerhalb einer Mauerstruktur und nach der Restaurierung. Foto: Fa. Ardis, Innsbruck; B. Nutz, Inst. f. Archäologien, Univ. Innsbruck.
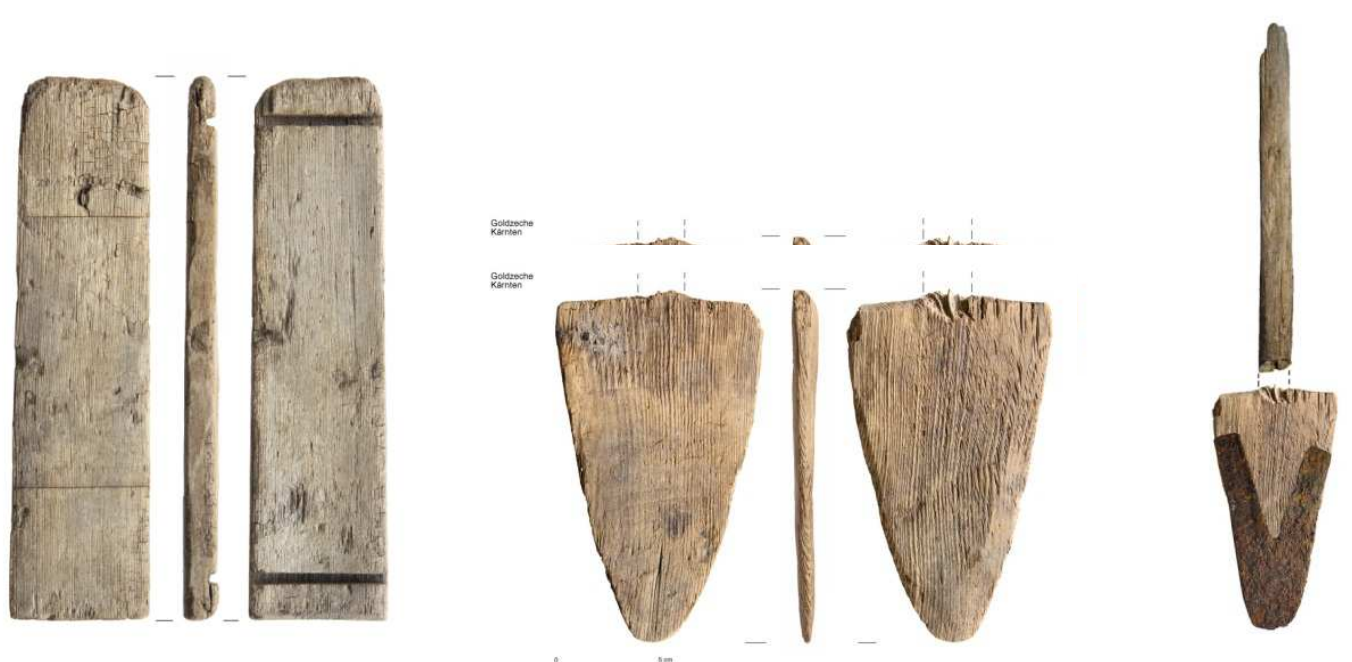

8

9

Abb. 8: Goldzeche, Heiligenblut, Kärnten. Daube von der Goldzeche. 9 Links: Hölzernes Spatenblatt. Rechts: Rekonstruktion. Foto: A. Blaickner, Inst. f. Archäologien, Univ. Innsbruck.

\subsection{Gletscherfundmeldungen}

\section{Seekarjoch, Pfunds, Ötztaler Alpen, Nordtirol}

Im Sommer 2016 entdeckte Univ. Prof. Dr. Klaus Oeggl am Seekarjoch, Gemeinde Pfunds, im Grenzbereich von Nordtirol und Südtirol, aber eindeutig auf österreichischer Seite, ein $107 \mathrm{~cm}$ langes Holzobjekt am Rand eines 2.845 m hochgelegenen Firnfeldes (Abb. 9). Der Fund wurde seinerseits umgehend Projektmitarbeitern des GAAA-Projektes zur Bestimmung vorgelegt. Eine entnommene Holzprobe datiert laut ${ }^{14} \mathrm{C}$-Untersuchung in das Hochmittelalter (MAMS 30885: 775+/-20 BP, cal AD 1223-1275, Wahrscheinlichkeit: 95,4\%). Das Artefakt weist zahlreiche Bearbeitungsspuren auf und erinnert im ersten Augenblick an ein Webschiff. Aufgrund der Länge von $107 \mathrm{~cm}$ muss dies aber als eher unwahrscheinlich gelten. Eine dendrochronologische Untersuchung wurde bereits in Auftrag gegeben. Die Auswertung ist noch ausständig. 


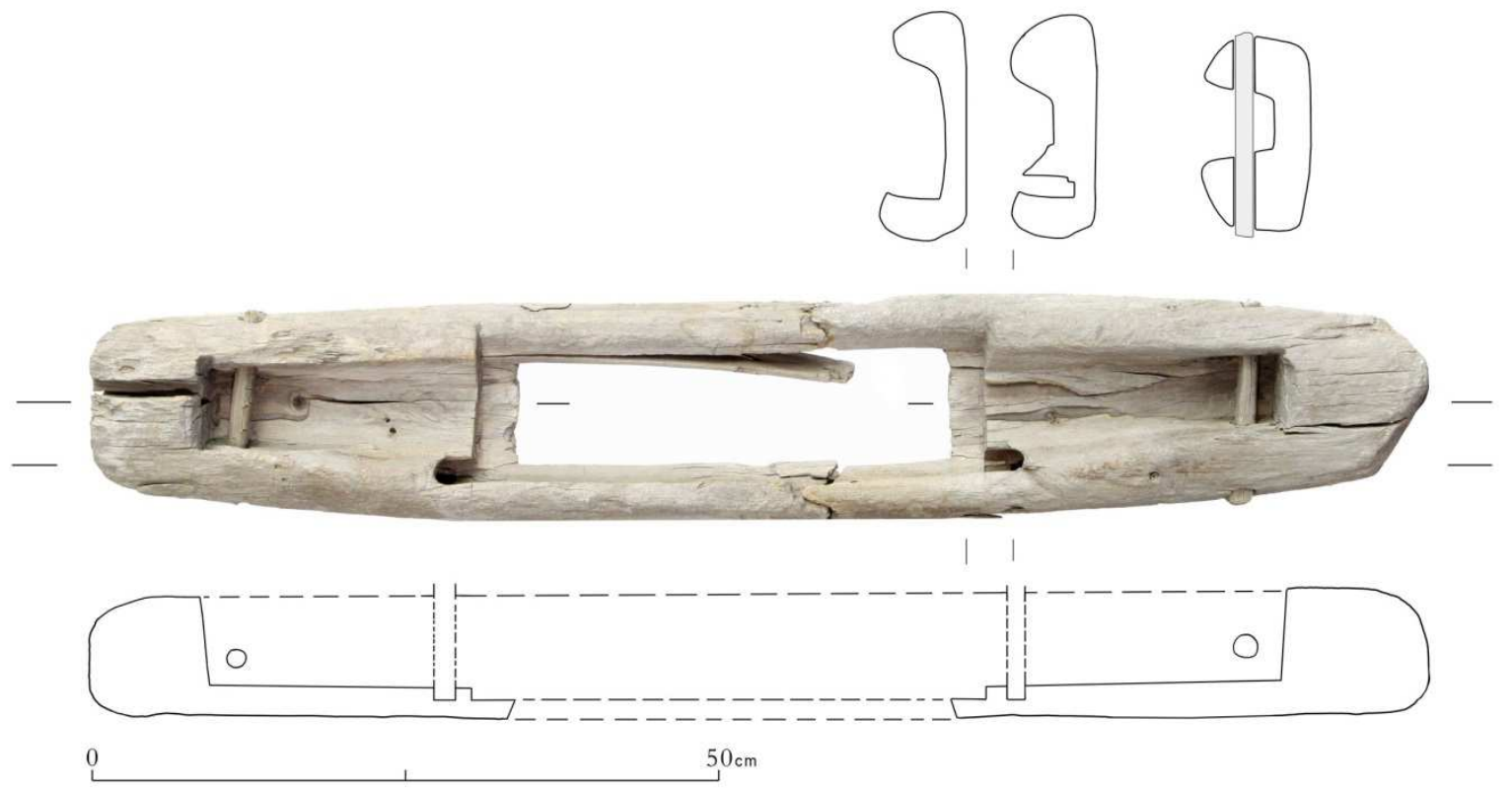

Abb. 10: Seekarjoch, Pfunds, Nordtirol. $107 \mathrm{~cm}$ langes bearbeitetes Holzobjekt aus dem Hochmittelalter. Foto/Zeichnung: A. Blaickner, Inst. f. Archäologien, Univ. Innsbruck.

\section{Schwarzensteinkees, Finkenberg, Zillertaler Alpen, Nordtirol}

Bereits am 13.10.2010 entdeckte der Mineraliensammler Walter Ungerank aus Aschau im Zillertal am Rand des Schwarzensteinkees im Zillertal auf 2.785 m Höhe zwei aus dem Eis ausgeaperte Artefakte, die er 2017 den Mitarbeitern des GAAA-Projekt übergab. Es handelt sich um ein Textilfragment und ein 22,5 cm langes Astfragment (Abb. 10). Das Textilfragment besteht aus (heute schwarz-brauner) Wolle und wurde mit z-gedrehtem Garn (Fadendurchmesser: 0,4 mm) in Fischgratköperbindung 2/2 mit 12 Fäden/cm gewebt. Auf Grund des in den Stoff eingesetzten metallenen Druckknopfes muss der Fund frühestens an den Beginn des 20. Jahrhunderts gestellt werden. Am 5. März 1885 reichte der Pforzheimer Heribert Bauer dazu ein Patent ein, aber erst 1903 kam der verbesserte Druckknopf von Hans Prym auf den Markt. Basierend auf dem recht feinen Wollstoff und dem Druckknopf könnte es sich hierbei um ein Unterwäsche-Fragment (alte Skiunterwäsche oder Ganzkörperunterwäsche, sog. "Long John") handeln. 


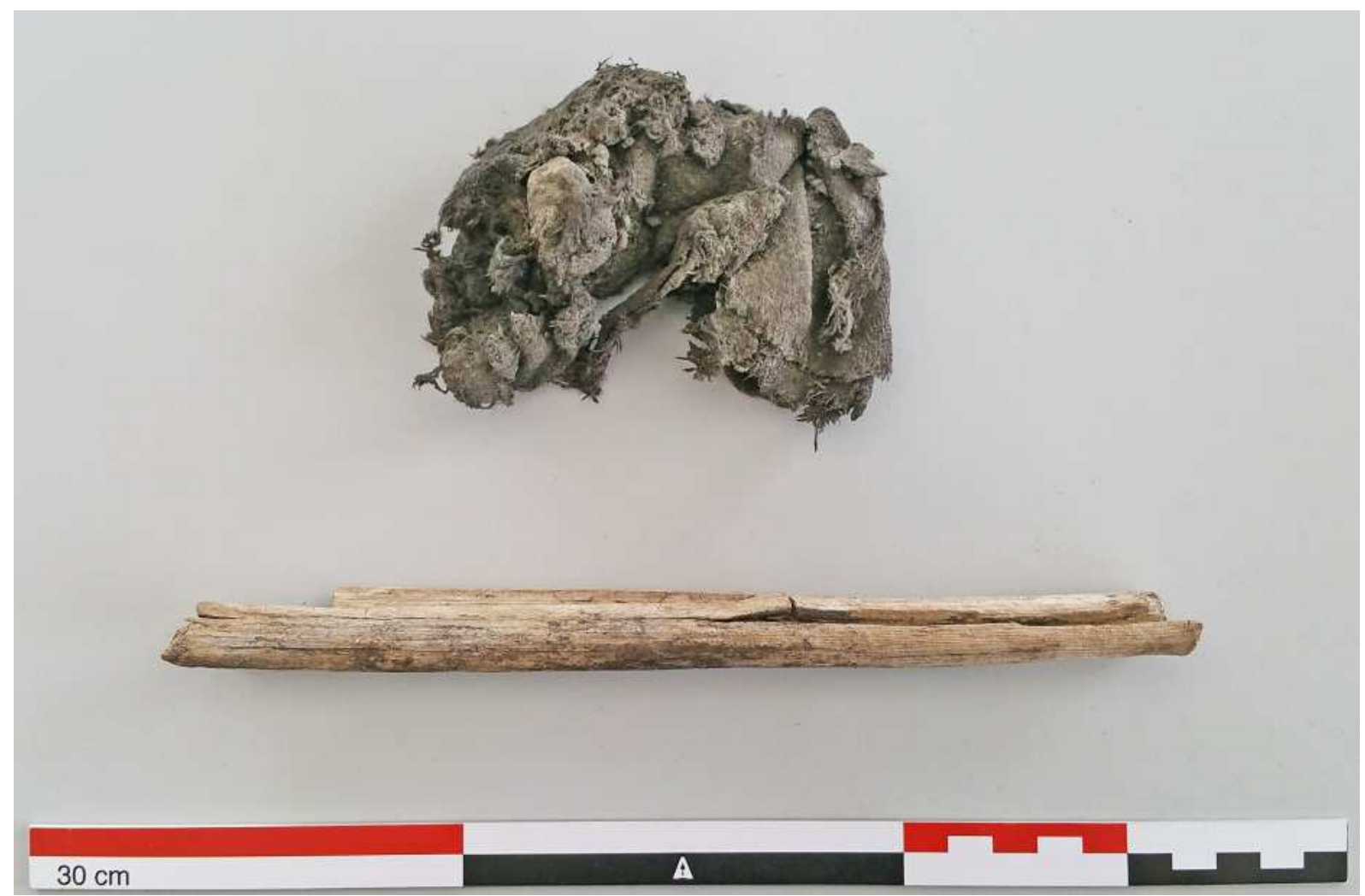

Abb. 10: Schwarzensteinkees, Zillertal, Nordtirol. Textilfragment und Holzobjekt nach Übergabe des Finders. Foto: T. Bachnetzer, Inst. f. Archäologien, Univ. Innsbruck.

\section{Bodenkar, Finkenberg, Tuxer Alpen, Zillertal}

Im Bereich des Bodenkars am Rand des Bodenkarkees auf rund $2800 \mathrm{~m}$ Höhe fand Roman Erler aus Tux im Zillertal ein Geweihfragment eines Hirsches, das laut ${ }^{14} \mathrm{C}$-Analyse ins 20. Jh. datiert. Das Stück stellt aufgrund fehlender eindeutiger Bearbeitungsspuren kein Artefakt dar (Abb. 11).

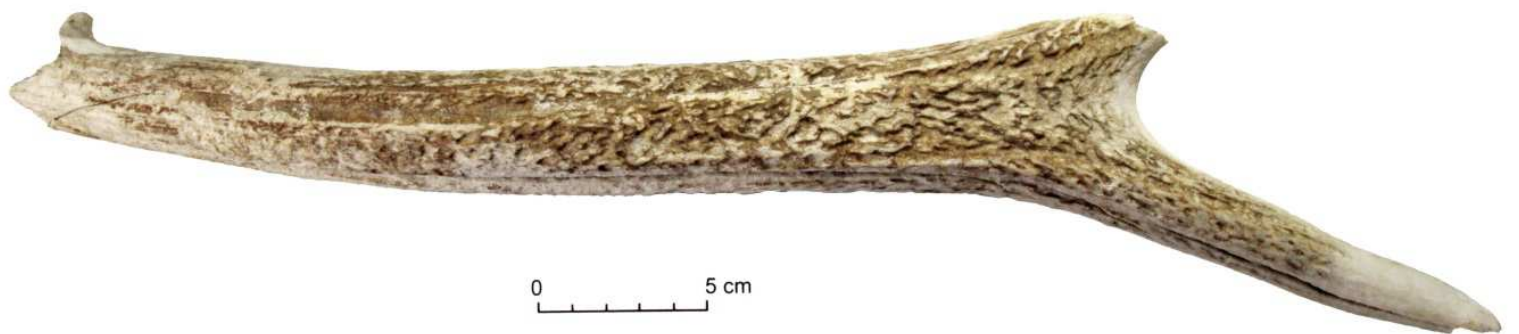

Abb. 11: Bodenkar, Zillertal, Nordtirol. Geweihfragment eines Hirsches vom. Foto: A. Blaickner, Inst. f. Archäologien, Univ. Innsbruck. 


\section{Predictive Modelling - Vorhersagemodell für gletscherarchäologische Funderwartungszonen}

Im Rahmen des Projektes sollte ein auf GIS basierendes Vorhersagemodell für gletscherarchäologische Funderwartungszonen erstellt werden. Dieses soll als Basis für gezielte Begehungen dienen, um eine maximale Zeitersparnis im Gelände zu ermöglichen. Unter Einbeziehung historischer und rezenter Gletscherstände werden Aussagen über zukünftige Gletscherbzw. Eisflächenrückgänge getroffen, die in Korrelation mit bisherigen Fundaufkommen gesetzt werden. Basierend auf den physischen Charakteristika des Terrains können hierdurch Regionen definiert werden, die ein ausnehmend hohes archäologisches Potenzial bieten bzw. besonders gefährdet sind. Das Modell ist noch in Ausarbeitung. Deshalb erfolgt die Endberichterstattung mit dem vorläufigen Zwischenergebnis.

\subsection{Ausgangssituation}

Basierend auf LiDAR-Daten und Orthofotos wurden die Ausmaße der österreichischen Gletscherstände errechnet, welche in den sog. Gletscherinventaren Österreichs (Austrian Glacier Inventory - AGI) resultierten (Abb. 12) ${ }^{8}$. Während der Kleinen Eiszeit (AGI LIA) nahmen die Gletscher Österreichs eine Fläche von ca. 941 km² ein. Im AGI 1 (1969) wurden 60\% dieser Fläche bedeckt, in AGI 2 (1998) $50 \%$ und $44 \%$ in AGI 3 (2004-2012).

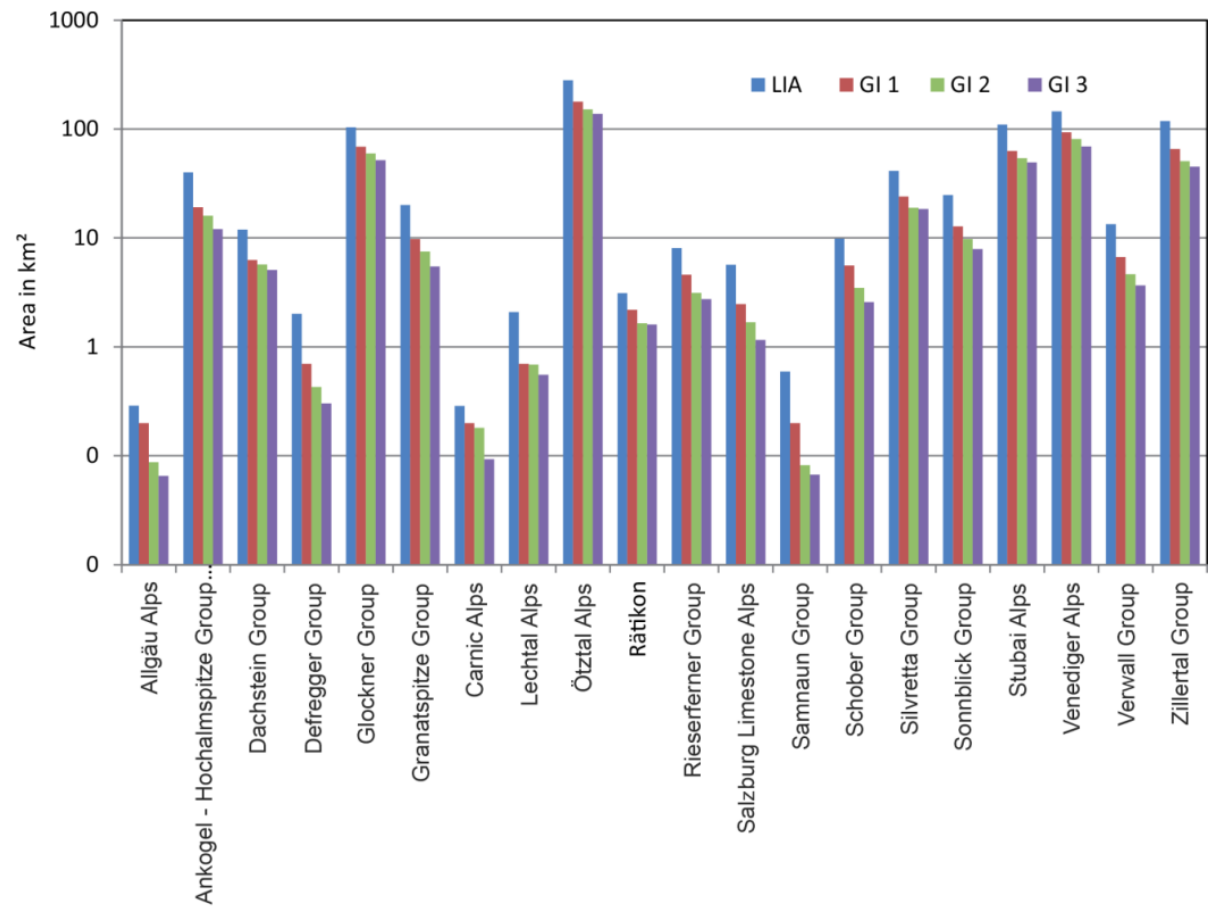

Abb. 12: Gletscherstände österreichischer Alpengruppen von AGI LIA bis AGI 3. Nach Fischer u. a. 2015, Abb. 9.

\footnotetext{
${ }^{8}$ Fischer et al. 2015.
} 
Der höchste absolute Gletscherrückgang zwischen AGI 2 und AGI 3 wurde in den Ötztaler Alpen, der Venediger Gruppe, Stubaier Alpen und der Glockner Gruppe verzeichnet. Diese Gebirgszüge nehmen etwa $75 \%$ der gesamten Gletscherfläche Österreichs ein (Abb. 13).

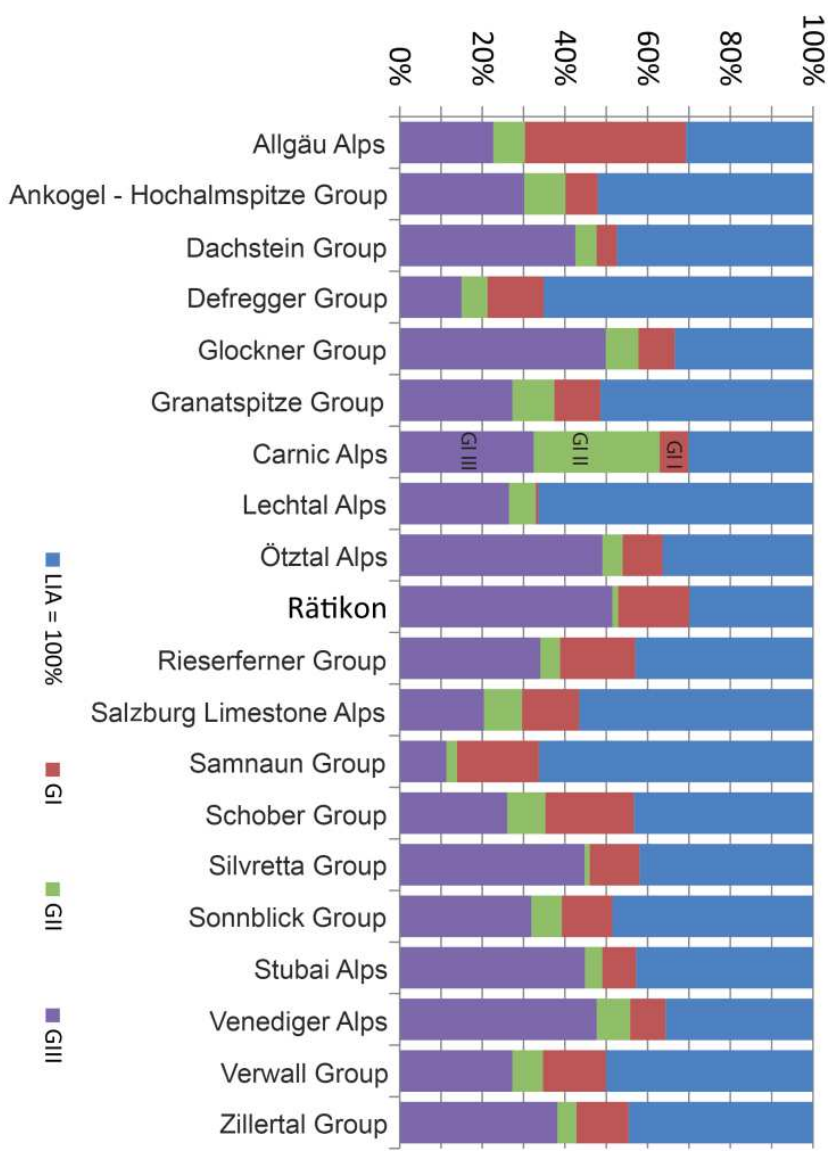

Abb. 13: Gletscherveränderungen österreichischer Alpengruppen in Prozent. nach Fischer u. a. 2015, Abb. 10.

Im Bewusstsein dieser dramatischen Veränderungen und erfolgter Berechnungen eines prognostizierten insgesamten Eisflächenrückgangs um $90 \%$ bis in das Jahr $2100^{9}$, gilt als vordringlichstes Ziel die systematische Detektion von Gebieten mit hohem gletscherarchäologischen Potenzial mithilfe GIS.

\subsection{Methodisches Vorgehen}

Im Rahmen des Projektes wurde ein methodisches Gesamtkonzept erarbeitet, dessen erste Schritte umgesetzt werden konnten und das Potenzial detaillierter weiterführender Studien in seiner Komplexität darstellt (Abb. 14).

\footnotetext{
${ }^{9}$ Rogers et al. 2014.
} 


\section{Predictive model}

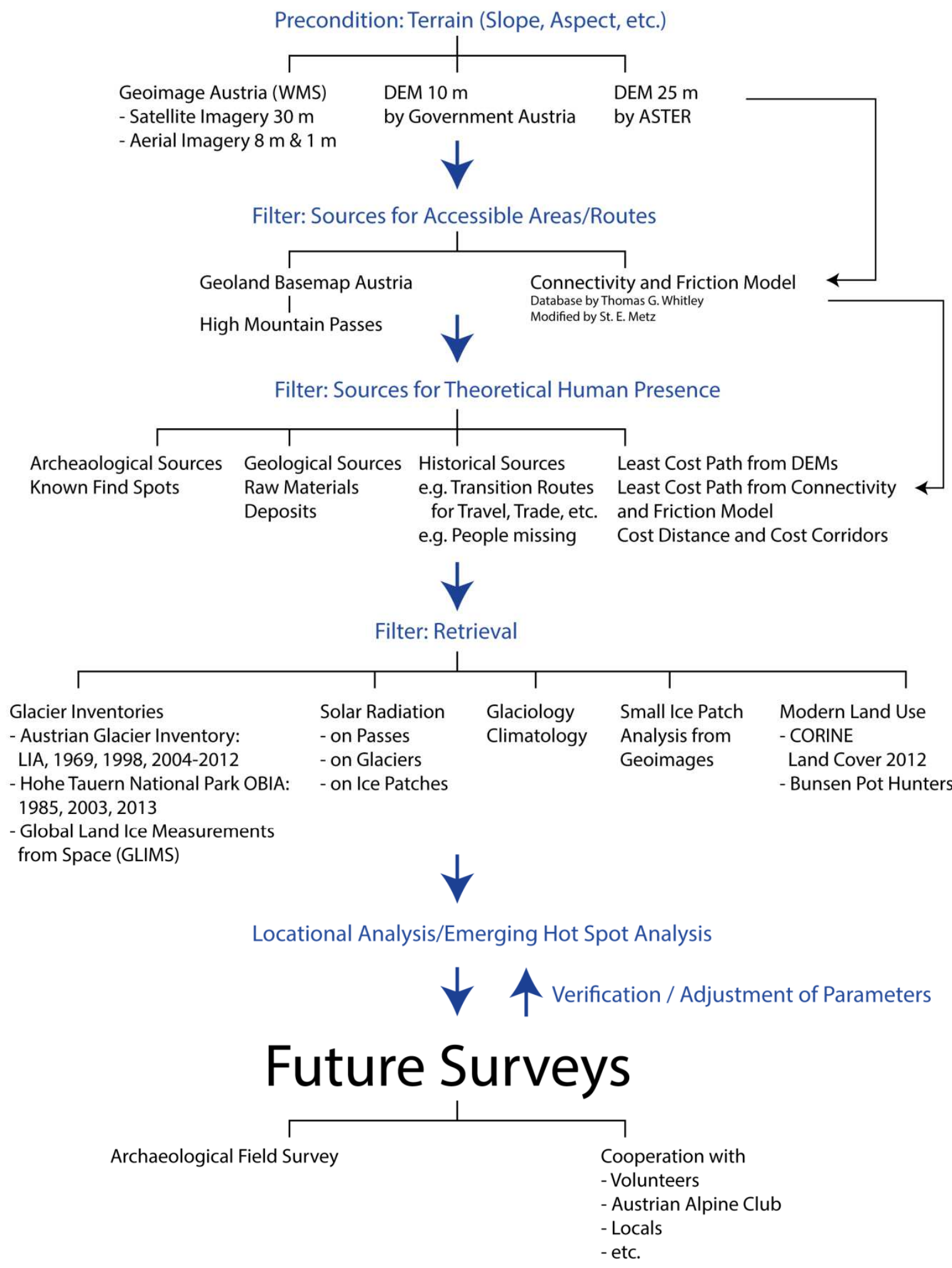

Abb. 14: Methodisches Gesamtkonzept eines auf GIS basierenden Vorhersagemodells. Grafik: Stephanie E. Metz, Inst. f. Archäologien, Univ. Innsbruck. 
Als Datengrundlage standen das kostenlose digitale Höhenmodell (DGM) Österreichs in $10 \mathrm{~m}$ Auflösung und das frei zugängliche Höhenmodell ASTER GDEM (Advanced Spaceborne Thermal Emission and Reflection Radiometer Global Digital Elevation Model) der Nasa zur Verfügung sowie die als WMS-Daten bereitgestellten Satelliten- und Luftbilder von Geoimage Austria. Aus den DGMs wurden die topografischen Parameter Hangneigung, - ausrichtung und Schummerung errechnet. Mittels Geoland Basemap Austria wurden hochalpine Passsituationen der Studiengebiete Ötztaler Alpen, Venediger Gruppe und Sonnblickgruppe herausgesucht und in das GIS integriert (Abb. 15).

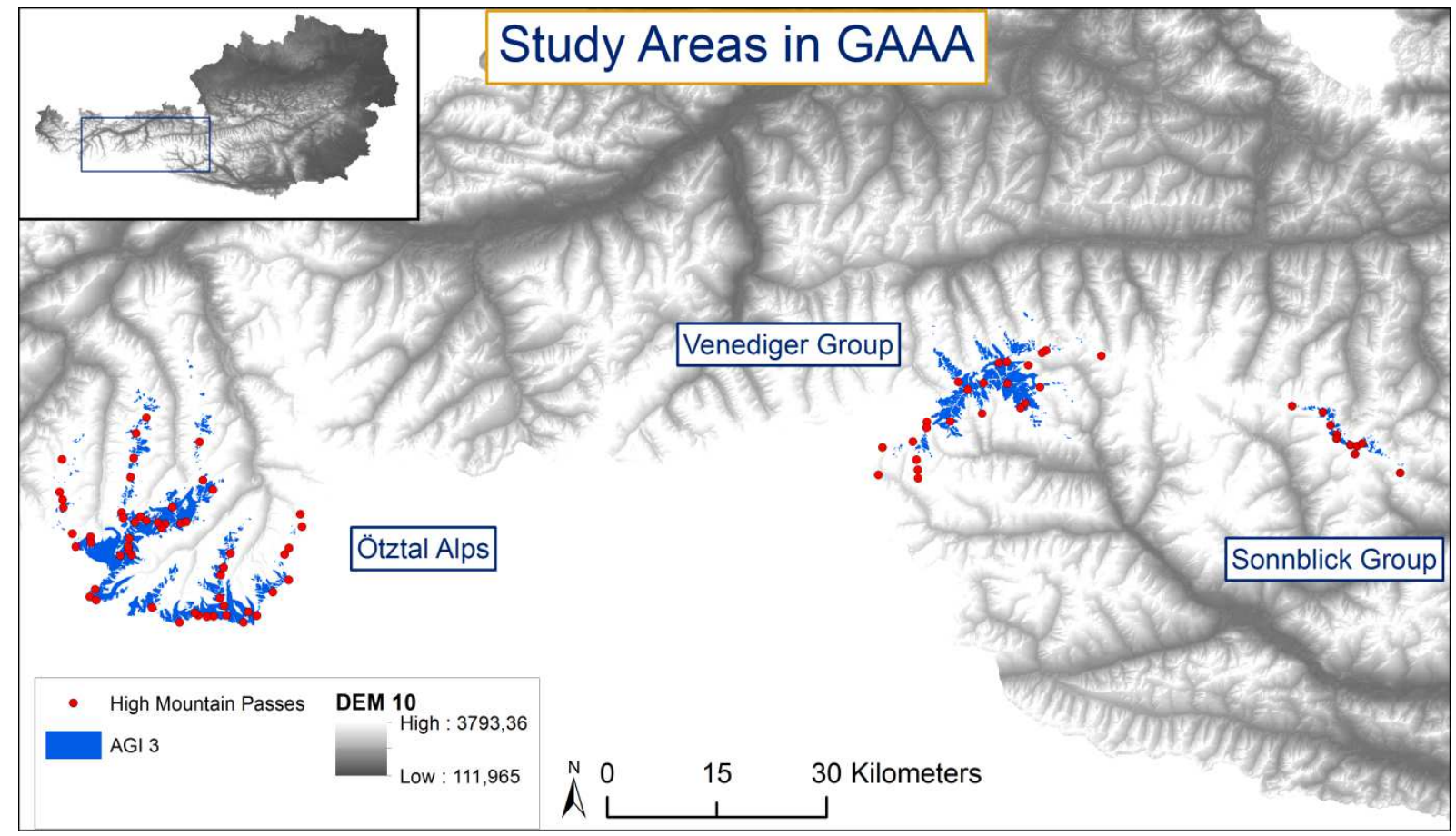

Abb. 15: Studiengebiet des Projektes GAAA, eingezeichnet AGI 3 und hochalpine Passsituationen. Grafik: Stephanie E. Metz, Inst. f. Archäologien, Univ. Innsbruck.

Um potenzielle Routen über die Alpen zu finden, fanden zwei Datensätze Verwendung, die freundlicherweise von Thomas G. Whitley, Sonoma State University, USA, zur Verfügung und weiteren Bearbeitung durch Stephanie E. Metz gestellt wurden. Dabei liegt im Folgenden die Idee zugrunde, potenzielle begehbare Routen zu finden, die nicht zu den in den jeweiligen Epochen bekannten Hauptverkehrsrouten gehören. Hierdurch sollen auch kleinere Wege detektiert werden, die nicht unter dem Einfluss von Autoritäten standen und somit beispielsweise für Schmuggler Alternativen für ihren Handel boten.

Das sog. Connectivity Model repräsentiert den Aufwand in Kalorien, der nötig ist, um mit einer Last von bis zu $25 \mathrm{~kg}$ zu Fuß zu reisen, basierend auf dem ASTER GDEM. Dem zugrunde liegt die Berechnung von kürzesten Wegen über die gesamte Alpenkette. Danach wurden Kostenkorridore analysiert, um die Werte zu finden, die zeigen, um wie viel sich Reisekosten erhöhen in Relation zu 
den kürzesten Wegen. Dieses Model zeigt, wie verbunden jeder einzelne Punkt zu einem anderen ist, wenn man als Ziel die Reise durch und über die Alpen annimmt. Dabei wird nicht nur die Hangneigung einbezogen, sondern auch die Nähe zu den besten Routen durch die Alpen. Dies bedeutet, dass flaches Terrain nahe oder mit einer guten Verbindung zwischen Norden und Süden der Alpen niedrig gewertet wird, hingehen dasselbe flache Terrain sehr weit von einer guten Routen entfernt höher gewertet wird. Steiles Terrain sehr weit von einer guten Route entfernt wird entsprechend am höchsten gewertet. Dabei wurden Hangneigungen von über 45 Grad ausgeschlossen, da diese für eine Begehung als zu steil angesehen werden. Dieses Modell ist als ein überregionales Modell anzusehen (Abb. 16).

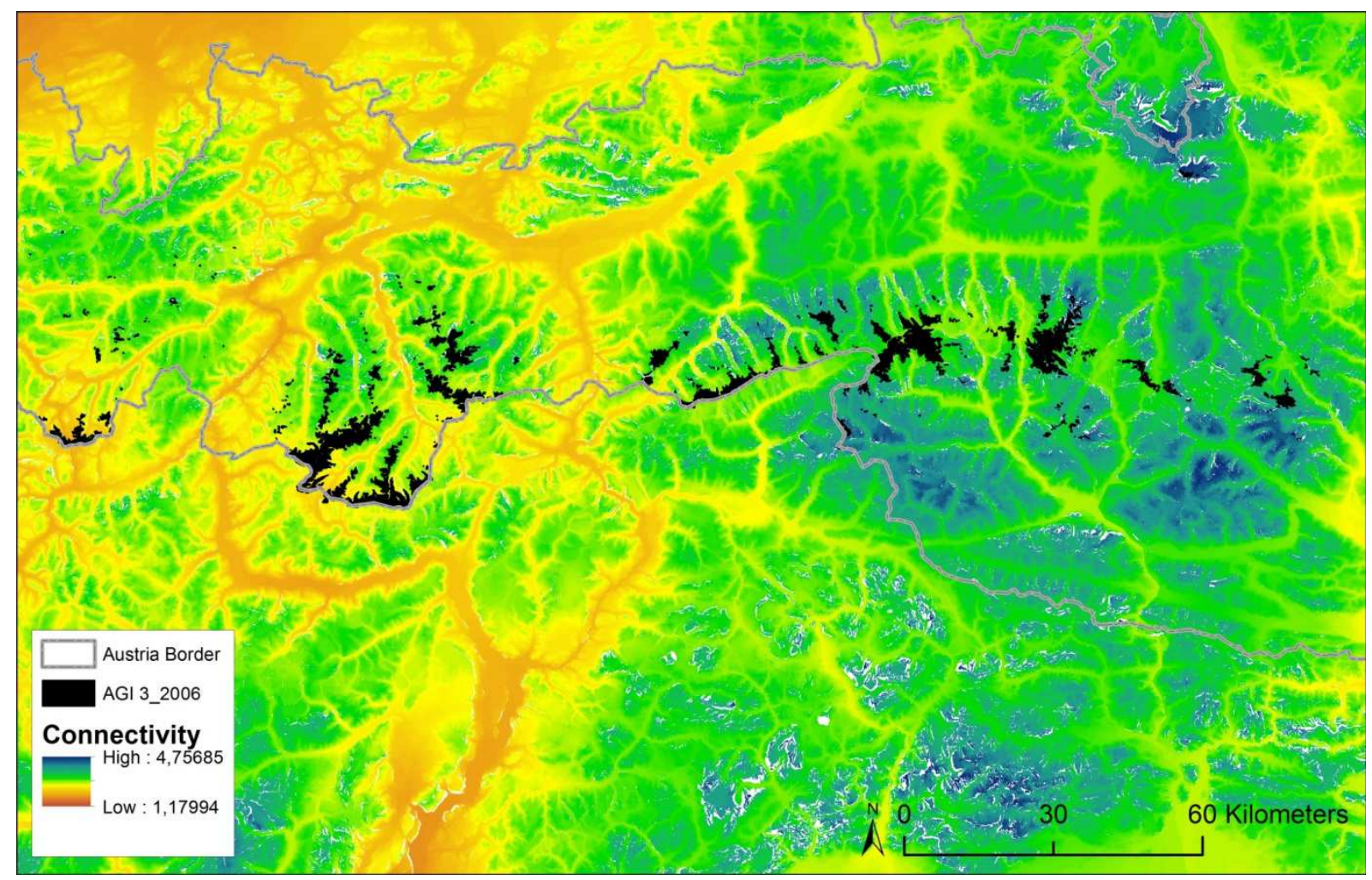

Abb. 16: Connectivity Model. Die hohen Werte repräsentieren hohe Kosten und somit die Regionen, die am wenigsten verbunden sind. Grafik: Stephanie E. Metz, Inst. f. Archäologien, Univ. Innsbruck.

Das sog. Friction Model, basierend auf ASTER GDEM, konvertiert Steigung in kalorische Kosten bezüglich Reisen zu Fuß. Die kalkulierten Werte beziehen sich auf die Anzahl der verbrauchten Kalorien pro Meter. Dieses Model kann nun verwendet werden, um Kostenentfernungen, Least Cost Path oder Kostenkorridore zu berechnen, deren Ergebnisse als verbrannte Kalorien pro Meter herausgegeben werden. Würde man beispielsweise eine Kostenentfernungsanalyse machen, würde bei flachem Terrain die Distanz mit 0,0675 multipliziert werden. Eine Distanz von 100 m würde 6,75 kcal ergeben - die Anzahl der verbrauchten Kalorien mit bis zu 25 kg Last und keiner Steigung. Entsprechend würde bei 45 Grad Steigung und einer Strecke von 100 m mit 4,0238 multipliziert werden, wodurch sich ein Aufwand von 402,38 kcal ergäbe. Hangneigungen von über 45 Grad 
wurden hier ebenfalls ausgeschlossen. Diese Kalorienwerte sind mit einer Reihe von verschiedenen Online-Quellen und Kalorienzähler kalibriert, um sicherzustellen, dass sie für eine durchschnittliche Größe geeignet sind. Einschränkend ist zu bemerken, dass dabei nicht die Kosten für das Gehen durch Wälder oder andere dichte Vegetation einkalkuliert ist. Dieses Modell ist als ein eher lokales Modell anzusehen (Abb. 17).

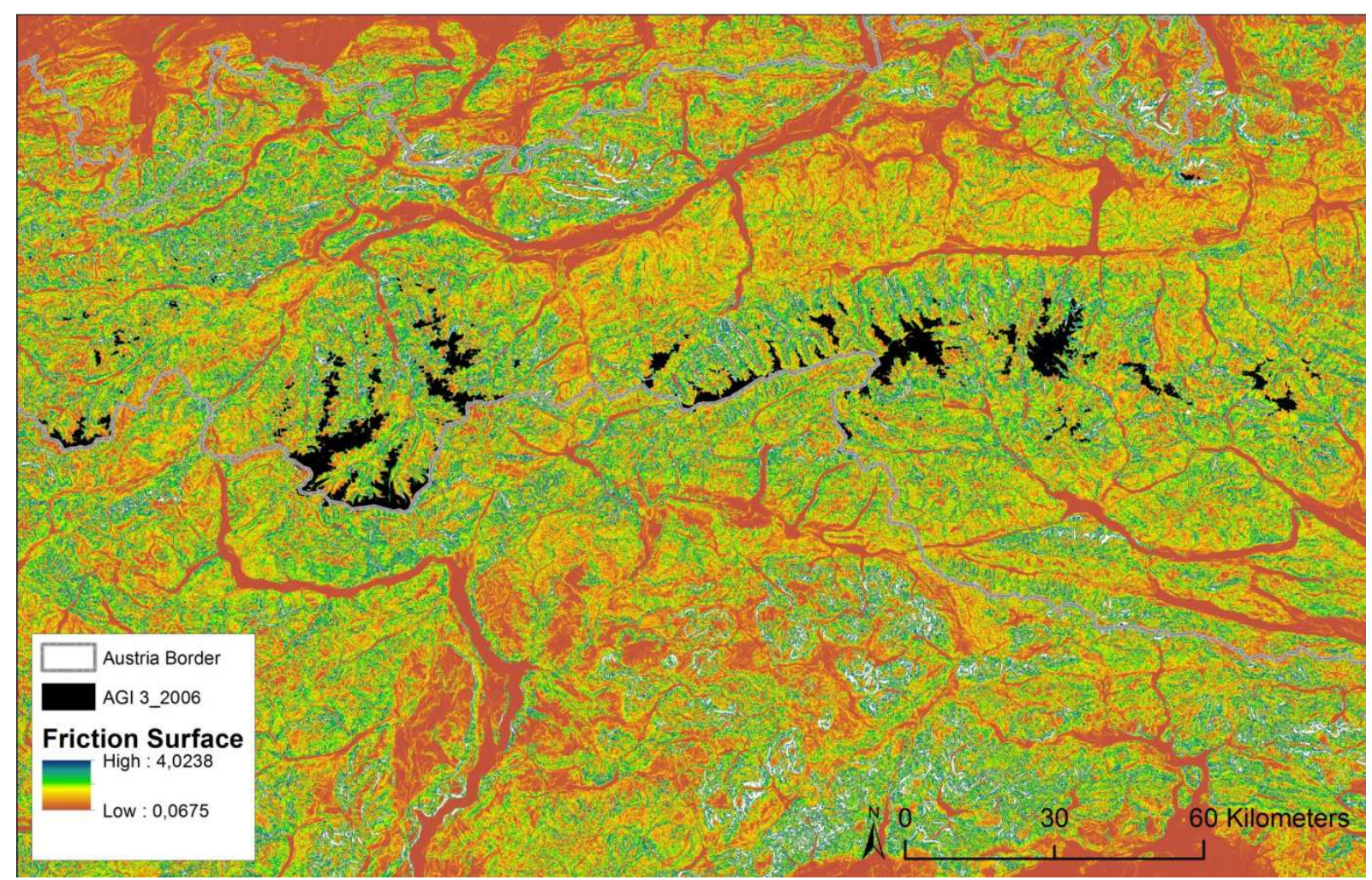

Abb. 17: Friction Model. Grafik: Stephanie E. Metz, Inst. f. Archäologien, Univ. Innsbruck.

Der Datenbestand bezüglich der Gletscherstände setzt sich zum einen aus denen der Österreichischen Gletscherinventaren 1969 (AGI 1), 1998 (AGI 2) und 2004-2012 (AGI 3) sowie Kleine Eiszeit (AGI LIA) zusammen. Zum anderen wurden freundlicherweise die durch Satellitenbildauswertungen generierten Gletscherausmaße im Gebiet des Nationalparks Hohe Tauern ${ }^{10}$ von B. Robson, University of Bergen, Norwegen, und D. Hölbling, Universität Salzburg, Österreich, zur Verfügung gestellt, wodurch für dieses Gebiet mit den Gletscherständen OBIA 1985, OBIA 2003 und OBIA 2013 eine höhere Datendichte erzielt werden konnte. Des Weiteren wurde der GLIMS (Global Land Ice Measurements from Space)-Datenbestand verwendet, um die Ausmaße der Gletscher über die Grenze hinweg nach Italien zu erhalten.

\footnotetext{
${ }^{10}$ Robson et al. 2016.
} 


\subsection{Fallstudie Venediger Gruppe - Umbaltal, Osttirol}

Das in diesem Projekt im besonderen Fokus archäologischer Prospektionen stehende Gebiet des Umbaltals in der Venediger Gruppe soll hier beispielhaft demonstrieren, welches Potenzial die Integration Geografischer Informationssysteme bietet.

Der Rückgang der Gletscherflächen der Venediger Gruppe wird mit 145,20 km² Fläche zur Kleinen Eiszeit um etwa zwei Drittel auf 55,31 km² Fläche im Jahr 2013 verzeichnet (Abb. 18).

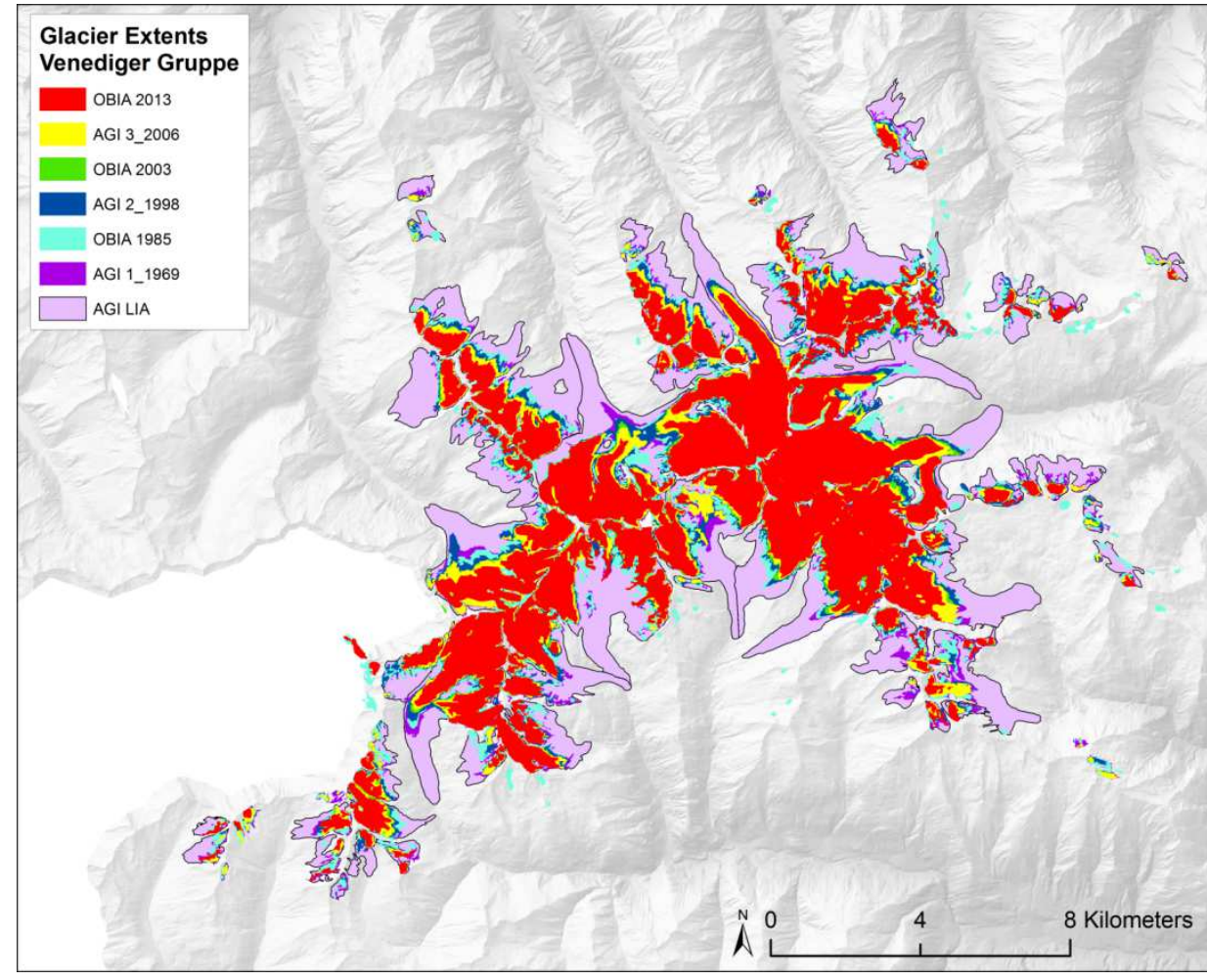

Abb. 18: Gletscherstände der Venediger Gruppe. Grafik: Stephanie E. Metz, Inst. f. Archäologien, Univ. Innsbruck.

\begin{tabular}{|l|l|}
\hline Glacier Extents & $\mathbf{K m}^{\mathbf{2}}$ \\
\hline AGI LIA & 145,20 \\
\hline AGI 1 & 93,44 \\
\hline AGI 2 & 81,01 \\
\hline AGI $3(2007 / 09)$ & 69,31 \\
\hline
\end{tabular}

OBIA 2013

Vergleicht man die Lage des Umbaltals mit den Übergängen Vorderes Umbaltörl und Hinteres Umbaltörl auf der Karte des Connectivity Modells, fällt auf, dass diese Region in der Gesamtbetrachtung weniger gut angebunden ist. Jedoch zeigt sich innerhalb dieses Gebiets, dass das Tal und die Pässe am besten geeignet sind, um nach Westen, Süd-Westen, Norden und Nord-Osten zu gelangen (Abb. 19). 

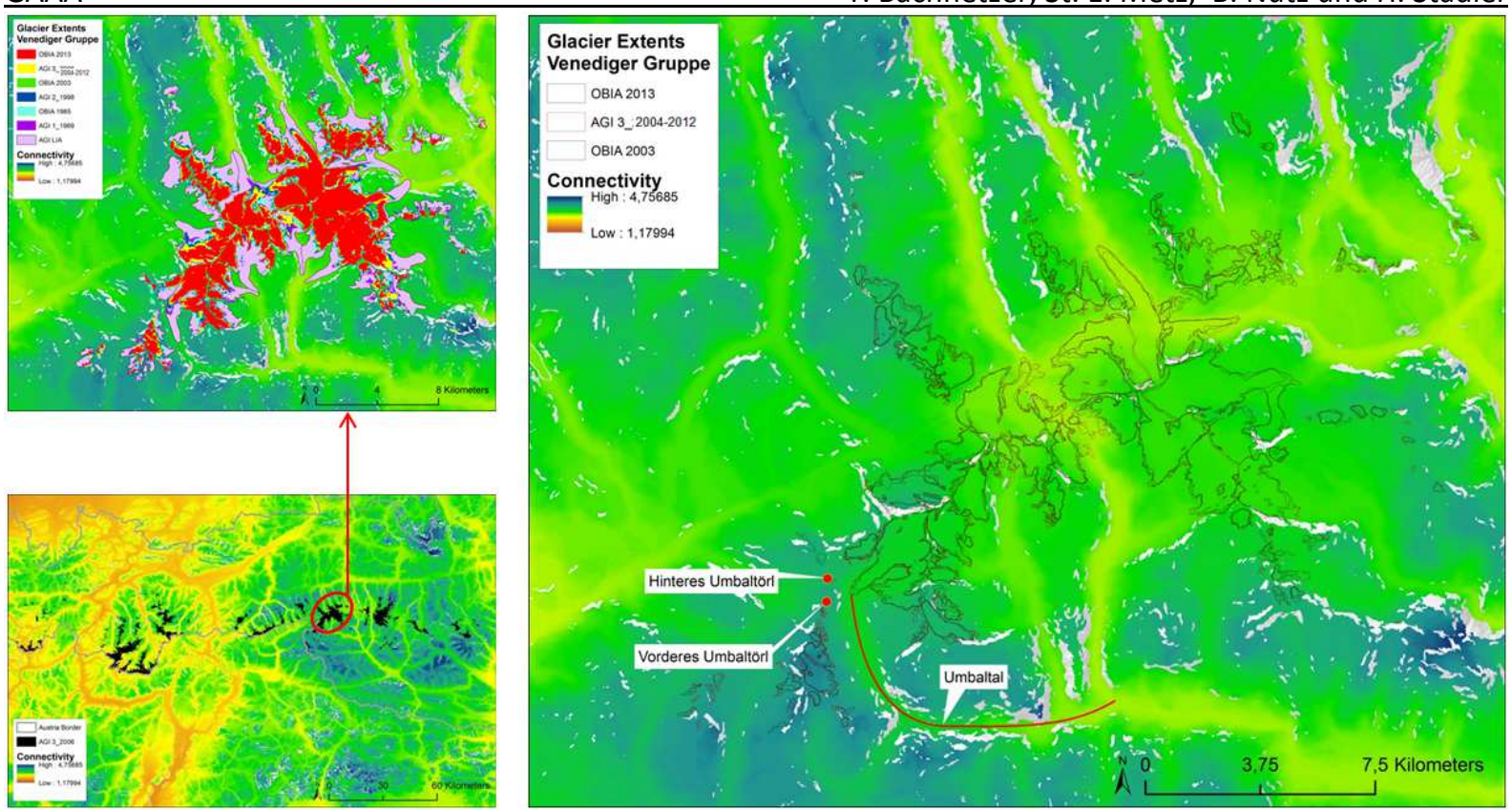

Abb. 19: links oben - Gletscherstände der Venediger Gruppe auf der Karte des Connectivity Modells; links unten - Connectivity Modell mit Venediger Gruppe hervorgehoben; rechts - Detaillausschnitt Umbaltal mit den Übergängen Vorderes Umbaltörl und Hinteres Umbaltörl. Grafik: Stephanie E. Metz, Inst. f. Archäologien, Univ. Innsbruck.

Gleiches zeigt sich für den aufzubringenden Aufwand, um dieses Gebiet zu durchqueren. Das Vordere und Hintere Umbaltörl gehören zu den kosteneffizientesten Übergängen dieser Region. Betrachtet man den rapiden Gletscherrückzug im Umbaltal, wird deutlich, dass dieses Tal mit seiner leichten Begehbarkeit sehr hohes archäologisches Potenzial bietet (Abb. 20).
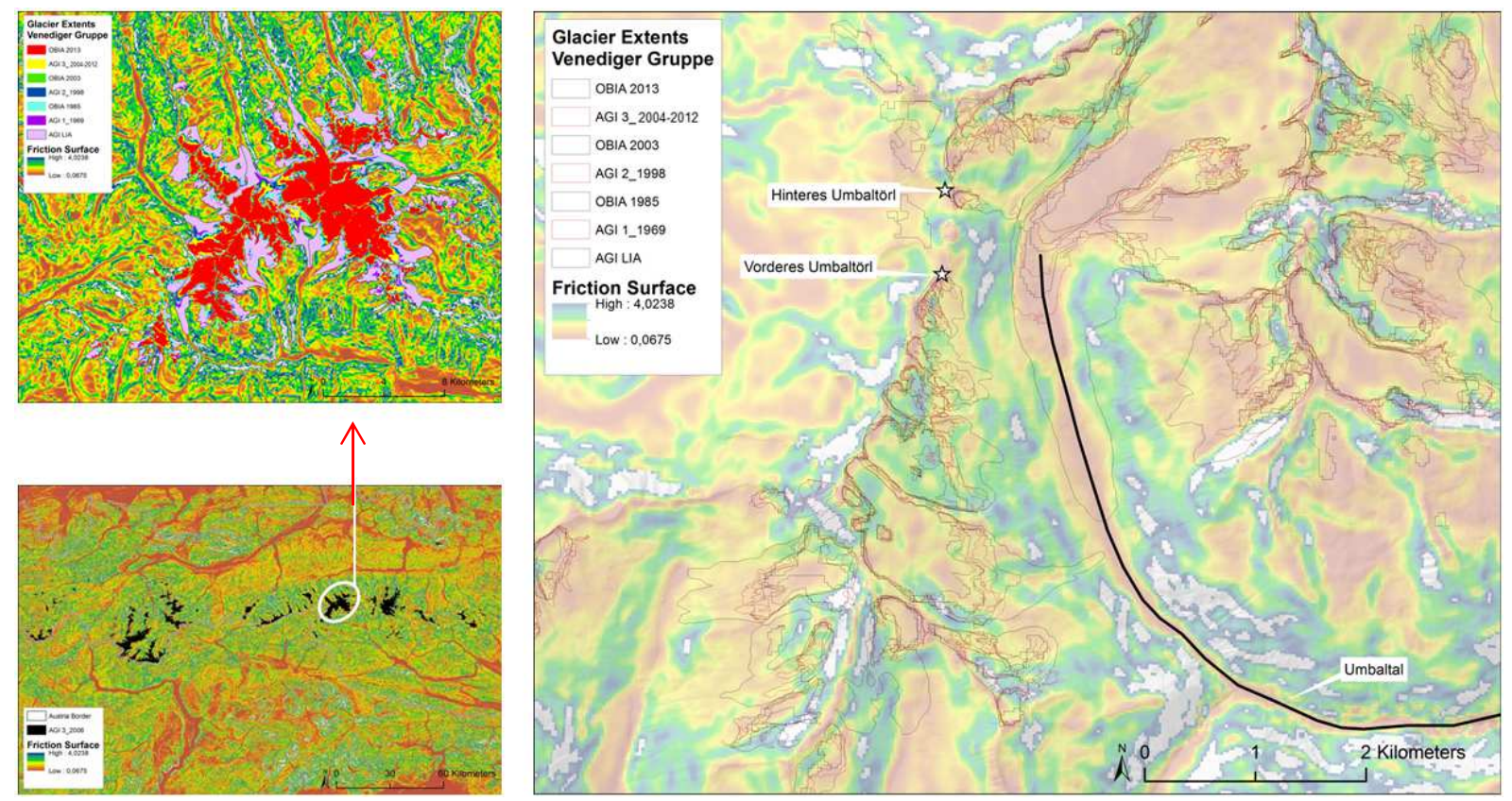

Abb. 20: links oben - Gletscherstände der Venediger Gruppe auf der Karte des Friction Modells; links unten Friction Modell mit Venediger Gruppe hervorgehoben; rechts - Detailausschnitt Umbaltal mit den Übergängen Vorderes Umbaltörl und Hinteres Umbaltörl und dem Rückzug der Gletscher. Grafik: Stephanie E. Metz, Inst. f. Archäologien, Univ. Innsbruck. 


\section{4 Öffentlichkeitsarbeit im Rahmen von Zeitungsartikeln, Flyern, Foldern und Homepage}

Gletscherfunde werden des Öfteren zufällig entdeckt. Umso wichtiger ist es die Allgemeinheit über den richtigen Umgang mit Gletscherfunden zu informieren und zuständige Anlaufstellen zu nennen. Dabei bildet die Öffentlichkeitsarbeit eine wichtige Säule, im Rahmen derer auf vielfältige Art und Weise über das Gletscherarchäologie-Projekt berichtet wird.

Im Zuge des vom BMWFW initiierten und finanzierten Zentrums für die Zusammenarbeit von Wissenschaft und Schule - Young Science wurde das Forschungsprojekt GAAA für einen Themenvorschlag für vorwissenschaftliche und Diplomarbeitsprojekte vorgeschlagen und auf deren Homepage veröffentlicht.

- Die Presse, Das eingefrorene Gedächtnis taut auf, 25.10.2016.

- Kleine Zeitung, Osttirol, Osttirols Gletscher als Fundgruben, 13.10.2016.

- Dolomiten, Innsbruck - Wenn die Vergangenheit ausapert, 13.10.2016.

- Tiroler Tageszeitung, Finger weg vom Fund im Eis, 13.10.2016.

- wissenswert, Magazin der Leopold-Franzens-Universität Innsbruck, Das wandernde Wesen Mensch, 11.10.2016.

- Die Presse, "Eisige Funde", 09.06.2017.

- Standort Tirol - "Von Schnee und Eis", 03.2016.

- zukunft forschung 1/16, Magazin für Wissenschaft und Forschung der Universität Innsbruck, Geschichten aus dem Eis.

- Land der Berge, "Tiefgekühlte Geschichte(n)", 5-6 2016

- Tiroler Tageszeitung, Lienz, Innsbruck - "Gletscherfunde im Visier von Experten aus aller Welt", 28.09.2015

- Tiroler Tageszeitung, Lienz, Innsbruck - "Augen auf am Gletscherrand", 14.07.2016

- Universität Innsbruck - zukunft forschung 01/16, „Geschichten aus dem Eis“, 08.06.2016

- Tiroler Tageszeitung, Sölden, Innsbruck, "Auch Bergwanderer halten Ausschau nach Ötzis Spuren", 27.05.2016

- Universität Innsbruck - Newsroom, „eschichten aus dem Eis“, 11.05.2016, (17.05.2016)

- Alpenverein, Bergauf 2/16, „Das ewige Eis, Ein Tresor für die Archäologie“, 09.04.2016

- Tiroler Tageszeitung Osttirol, "Gletscherfund gibt Rätsel auf", 10.02.2016

- die Presse, "Der verborgene Schatz der alpinen Gletscher", 29.01.2016

- Die bereits im 1. und 2. Zwischenbericht erwähnten Flyer über das richtige Verhalten im Falle eines getätigten archäologischen Gletscherfundes sind fertiggestellt und auf vielen Hütten 
verteilt sowie auf der GAAA-Homepage zum Download bereitgestellt (Abb. 20). Kooperationspartner: Bundesdenkmalamt Österreich (BDA), Österreichischer Alpenverein ÖAV).

- Informationen über das Projekt sind laufend über die GAAA-Homepage aktualisiert worden.

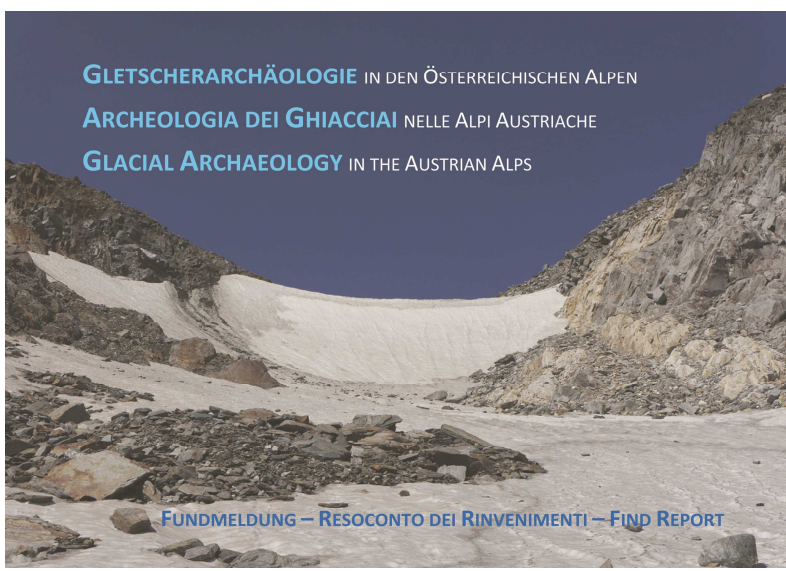

Etwas im Eis Gefunden? Trovato gualcosa nel Ghiaccio? Found Something in the ICE? VORGEHENSWEISE: FUND FOTOGRAFEREN - FUNDORT MARKLEREN -ZUSTÄNOIGE STEUE INFORMIEREN

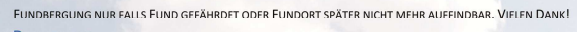

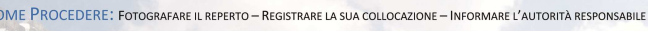

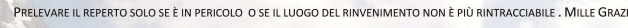
RECOVER FIND ONIY IF CONSERVATION IS NOT GUARANTEED OR IF LOCATION MAY NOT BE FOUND AGAIN. THANSS! FUNDMATERIALEN (BEISPIELE): HOLZ-LEDER-FEL-KNOCHEN-METAL-STEIN-TEXTIEN

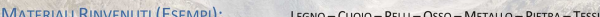

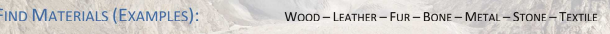
Universitat Insbruck- - nstitut fur Archäalogien - Langer Weg $11-6020$ innsbruck

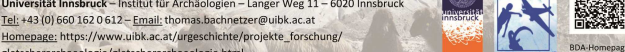

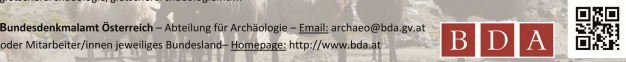

Abb. 20: Gletscherarchäologieflyer. T. Bachnetzer, Inst. f. Archäologien, Univ. Innsbruck.

\section{Tagung, Workshop und Ausstellung / Prospektionen in angrenzenden Gebieten}

Im Rahmen des GAAA-Projektes fanden in Innsbruck 2016 der vom ÖAW mitfinanzierte internationale Gletscherarchäologiekongress Frozen Pasts 4 sowie 2015 ein GletscherarchäologieWorkshop statt. Aufgrund der im Oktober 2016 durchgeführten Gletscherarchäologietagung haben sich die GAAA-Mitarbeiter dazu entschlossen den zweiten Workshop am Ende des Projektes im Frühjahr 2017 wegen der zeitlichen Nähe nicht abzuhalten, da alle potentiellen Workshopteilnehmer und Workshopteilnehmerinnen auf der Tagung vertreten waren und allfälliges dort ausführlich besprochen wurde.

\subsection{Gletscherarchäologie-Tagung Frozen Pasts 4 (Innsbruck, 12.-16.10.2016)}

An der Organisation der Tagung waren Gletscherarchäologen aus Österreich, der Schweiz und aus Italien beteiligt. Mit Wissenschaftlern und Wissenschaftlerinnen aus 11 Nationen, die in insgesamt 45 Vorträgen und 9 Postern ihre bislang erzielten Untersuchungsergebnisse und Ausblicke für zukünftige Forschungen präsentierten, konnten weitreichende und wertvolle Kooperationen geknüpft werden. Zudem wurde eine Gletscherarchäologieausstellung konzipiert, in der Gletscherfunde aus Österreich und der Schweiz präsentiert wurden (Abb. 21). 


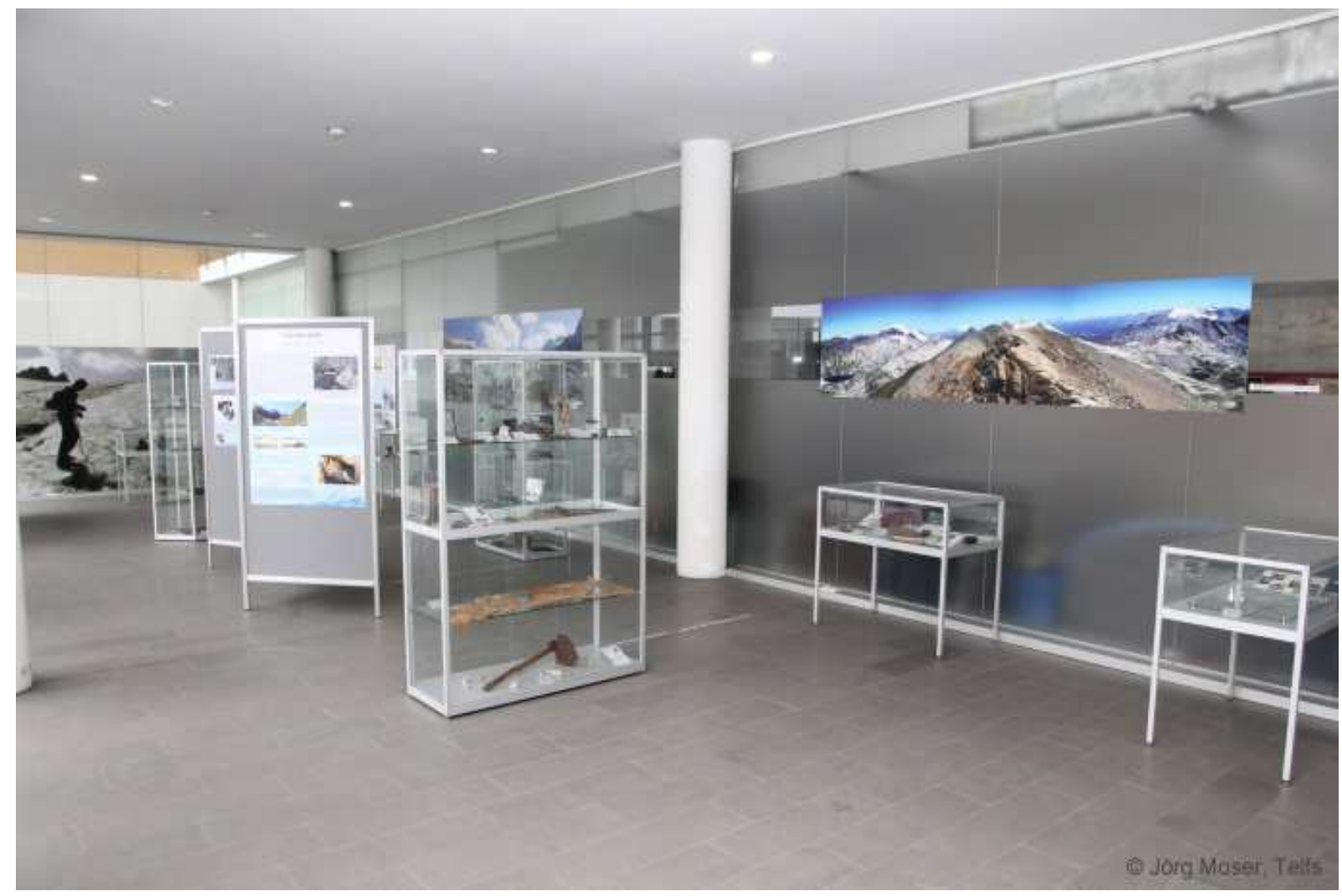

Abb. 21: Gletscherarchäologieausstellung, Zentrum für alte Kulturen, Univ. Innsbruck. Foto: J. Moser, Inst. f. Archäologien, Univ. Innsbruck.

\subsection{Gletscherarchäologie-Workshop (Innsbruck, 22.12.2015)}

Die Gletscherregionen im Westen Österreichs reichen im Süden (Tirol) in die autonome Provinz Bozen/Südtirol/Italien und im Westen (Vorarlberg) in den Kanton Graubünden/Schweiz über die Bundesgrenzen hinaus. Im Rahmen des Workshops wurden die Kontakte zu Forschern aus der Schweiz (Archäologischer Dienst Graubünden, kAltes Eis) und Südtirol (Bundesdenkmalamt, Abteilung Archäologie) intensiviert, laufende Untersuchungen präsentiert und mögliche zukünftige Forschungskooperationen besprochen.

\subsection{Prospektionen Graubünden/Südtirol}

In der Folge des Workshops ergab sich für GAAA-Mitarbeiter die Möglichkeit bei gletscherarchäologischen Geländebegehungen in Graubünden und Südtirol mitzuwirken. Mit dem archäologischen Dienst Graubünden (Thomas Reitmaier) wurde eine aus dem Gletscher ausgeaperte mesolithische Bergkristallabbaustelle auf 2800 m Höhe prospektiert. Die Prospektion wurde vom RTR (Schweizer Fernsehsender) begleitet und in eine Dokumentation integriert ${ }^{11}$. Mit dem Amt für

\footnotetext{
${ }^{11}$ Reitmaier et al. 2016.
} 
Denkmalpflege der autonomen Provinz Bozen/Südtirol (Hubert Steiner) die prähistorische Gletscherfundstelle am Langgrubenjoch prospektiert ${ }^{12}$.

\section{Vortragstätigkeit und Posterpräsentationen bei Tagungen}

Insgesamt wurden innerhalb der Projektlaufzeit zehn Vorträge und drei Posterpräsentationen im Inund Ausland gehalten.

\subsection{Vorträge}

- Vortragende/r: Bachnetzer, Thomas, Co-Autoren und Co-Autorinnen: Stadler, Harald; Metz, Stefanie: Gletscherarchäologie in den österreichischen Alpen - Bilanz 2016, Tag des Institutes für Archäologien - Bilanz 2015/2016, Innsbruck, 13.01.2017.

- Vortragender: Stadler, Harald, Notlandungen und Flugzeugcrashs in Gletschern der Tiroler Alpen. 3. Montafoner Gipfeltreffen, Schruns, 18.-22.10.2016.

- Vortragender: Bachnetzer, Thomas, Co-Autoren und Co-Autorinnen: Metz, Stephanie; Stadler Harald: Glacial Archaeology in the Austrian Alps (GAAA) - Past - Present - Future, Frozen Pasts 4, Innsbruck, 12.-16.10.2016, Session: Glacial Archaeology - finds \& sites, methods, surveys $\&$ analyses.

- Vortragender: Stadler, Harald: Anstelle eines Schlusswortes: Migrationsbefunde im 21. Jahrhundert. 25 Jahre Ötzi: Der Mann im Eis und die Migration: ein zentrales Phänomen der Menschheitsgeschichte, Innsbruck, 08.10.2016.

- Vortragende: Metz, Stephanie, Co-Autoren: Bachnetzer, Thomas; Stadler Harald: Glacial Archaeology in the Austrian Alps (GAAA), CAA Conference Oslo, 29.03-02.04.2016, Session: Using GIS Modeling to Solve Real-World Archaeological Problems

- Vortragender: Bachnetzer, Thomas, Co-Autoren: Flatscher, Elias; Stadler, Harald: Gletscherarchäologische Prospektionen im Umbaltal, Osttirol. beFUNDet 2015: Archäologischer Jahresrückblick für Tirol und Vorarlberg, Innsbruck, 05.02.2016.

- Vortragender: Stadler, Harald: Aktuelles aus der Gletscherarchäologieforschung in Österreich. Workshop Gletscherarchäologie in den Österreichischen Alpen, Innsbruck, 22.12.2015.

\footnotetext{
${ }^{12}$ Steiner 2015. - Steiner et al. 2016.
} 
- Vortragender: Bachnetzer, Thomas, Co-Autor: Stadler, Harald: Glacier Archaeology in the Austrian Alps (GAAA) - Prospektionen Umbaltal. Workshop Gletscherarchäologie in den Österreichischen Alpen, Innsbruck, 22.12.2015.

- Vortragende: Stadler, Harald; Bachnetzer, Thomas: Gletscherarchäologie und Hochgebirgsarchäologie in den Österreichischen Alpen, Alpenverein Österreich, Innsbruck, 02.12.2015.

- Vortragende: Nutz, Beatrix; Bachnetzer, Thomas: Ersatz für das Goldene Vlies - der Einsatz von Textilien und die Bekleidung des Bergmannes im spätmittelalterlichen und frühneuzeitlichen Goldbergbau. 10. HiMAT Milestone Meeting, Innsbruck, 06.11.2015.

\subsection{Poster}

- Vortragende: Metz, Stephanie E., Co-Autoren: Bachnetzer, Thomas; Stadler, Harald: Gletscherarchäologie in den Österreichischen Alpen - Ein Konzept, das Unaufhaltsame zu greifen, Session AG CAA Computeranwendungen und Quantitative Methoden in der Archäologie, 8. Workshop, Mainz 2017.

- Vortragende: Metz, Stephanie E. Co-Autoren: Bachnetzer, Thomas, Stadler, Harald: Glacial Archaeology in the Austrian Alps (GAAA) - Tracing the Disappearing Using GIS. 4th International Glacial Archaeology Symposium: Frozen Pasts, Innsbruck, 14.10.2016.

- Vortragende: Metz, Stephanie, Co-Autoren: Stadler Harald; Bachnetzer, Thomas; Glacial Archaeology in the Austrian Alps (GAAA), CAA Conference Oslo, 29.03-02.04.2016, Session: Using GIS Modeling to Solve Real-World Archaeological Problems.

\section{Publikationen}

Die Ergebnisse der gletscherarchäologischen Forschungen werden jährlich in den Fundberichten aus Österreich ausführlich dargelegt. Des Weiteren werden die Ergebnisse der Untersuchungen im Rahmen des bereits vom 12.-16.10. ausgerichteten internationalen Gletscherarchäologiesymposiums Frozen Pasts im Journal of Glacial Archaeology Vol. 3 publiziert.

- Bachnetzer, Thomas; Nutz, Beatrix; Stadler, Harald; Metz, Stephanie E. (2017): Glacial Archaeology in the Austrian Alps. Past - Present - Future. In: Journal of Glacial Archaeology Vol. 3 (in Druck).

- Bachnetzer, Thomas; Stadler, Harald (2018): Bericht zu den gletscherarchäologischen Untersuchungen des Instituts für Archäologien der Universität Innsbruck im Umbaltal, Virgental, Osttirol, 2015 (in Druck). 
- Bachnetzer, Thomas; Flatscher, Elias; Stadler, Harald (2017): Bericht zu den gletscherarchäologischen Untersuchungen des Instituts für Archäologien der Universität Innsbruck im Umbaltal, Virgental, Osttirol, 2015. Fundber. Österreich 54/2015, D6630D6639; 400-401.

- Nutz, Beatrix (2016): Spätmittelalterliche und neuzeitliche Textilien aus Goldbergbaugebieten in Österreich. Fundber. Österreich 53, S. 111 - 122.

- Bachnetzer, Thomas; Stadler, Harald: Das ewige Eis. Ein Tresor für die Archäologie, In: Bergauf, Mitgliedermagazin des Österreichischen Alpenvereins vom 09.04.2016, 26-29.

- Nutz, Beatrix; Bachnetzer, Thomas; Stadler, Harald: Tiefgekühlte Geschichte(n). Was Gletscher freigeben. In: Land der Berge. Österreichs Outdoor-Magazin vom 15.09.2016, 3438.

\section{Dank}

Die Berichterstatter bedanken sich recht herzlich bei der Österreichischen Akademie der Wissenschaften für die zur Verfügung gestellten finanziellen Mittel für das Projekt "Glacial Archaeology in the Austrian Alps (GAAA)“.

\section{Im Text erwähnte Literatur}

Bachnetzer et al. 2017

T. Bachnetzer/ E. Flatscher/ H. Stadler, KG Prägraten am Großvenediger, OG Prägraten am Großvenediger. Fundber. Österreich 54, 2015, 2017, 400-401; Bericht zu den gletscherarchäologischen Untersuchungen des Instituts für Archäologien der Universität Innsbruck im Umbaltal, Virgental, Osttirol, 2015, D6630-D6639.

Battisti 1934

C. Battisti, L'Etrusco e le altre lingue preindoeuropee d'Italia, Studi Etruschi 8, 1934, 179-196.

Bodner 2003

R. Bodner, Die Opferwidder-Prozession nach Lavant und Obermauern. In: Gemeinde Virgen, Pfarre Virgen (Hrsg.), Die Kirche zu Unser Lieben Frau, Maria Schnee, Obermauern in Virgen, 2003, 29-37.

R. Cornioley, Der Flugzeugabsturz einer amerikanischen Dakota auf dem Gauligletscher im November 1946. Berner Zeitschrift für Geschichte 3, 2006, 115-155. 
Fischer u. a. 2015

A. Fischer/ B. Seiser, M. Stocker Waldhuber/ C. Mitterer/ J. Abermann, Tracing glacier changes in Austria from the Little Ice Age to present using lidar-based high-resolution glacier inventory in Austria. The Cryosphere 9, 2015, 753-766.

Ghislanzoni 1928

Ghislanzoni, Ettore, Collalbo-Stazione preistorica. Notizie degli Scavi di Antichità comunicate all'Accademia dei Lincei, Serie VI, Vol. IV, 1928, 294-323.

Mayr 1958

K. M. Mayr, Die Birkenrute von Klobenstein am Ritten und ihre Inschrift. Ein Deutungsversuch. Der Schlern 3/4, 1958, 151-153.

Nutz 2015

B. Nutz, Mining for Textiles - Textiles for Mining. Preliminary Report on Textiles from Gold Mining Sites in Austria. In: K. Grömer/ F. Pritchard (Hrsg.), Aspects of the design, production and use of textiles and clothing from the Bronze Age to the early modern era: NESAT XII : the North European Symposium of Archaeological Textiles 21st-24th May in Hallstatt, Austria. Archaeolingua 33.

Archaeological Alapítvány (Budapest 2015) 25-41.

Nutz 2016

B. Nutz, Spätmittelalterliche und neuzeitliche Textilien aus Goldbergbaugebieten in Österreich. Fundberichte aus Österrreich 53/2014, 2016, 111-122.

Robson u. a. 2016

B. A. Robson/ D. Hölbling/ Ch. Nuth/ T. Strozzi/ S. O. Dahl, Decadel Scale Changes in Glacier Area in the Hohe Tauern National Park (Austria) Determined by Object-Based Image Analysis. Remote Sensing 8, 67, 2016. doi: 10.3390/rs8010067

Rogers u. a. 2014

St. R. Rogers/ M. Fischer/ M. Huss, Combining glaciological and archaeological methods for gauging glacial archaeological potential. Journal of Archaeological Science 52, 2014, 410-420.

Steiner et al. 2016

H. Steiner/ R. Gietl/ A. Bezzi/ G. Naponiello/ K. Nicolussi/ T. Pichler, Gletscherfunde am Langgrubenjoch (Gde. Mals und Gde. Schnals) in Südtirol. Vorbericht. Archäologisches Korrespondenzblatt 46/2016(2), 2016, 167-182.

\section{Steiner 2015}

H. Steiner, Aufgetaut - Gletscherfunde am Langgrubenjoch (Matsch / Schnalstal). In: P. Gleirscher/ L. Andergassen (Hrsg.), Antiquitates Tyrolenses. Festschrift für Hans Nothdurfter zum 75. Geburtstag. Veröff. Südtiroler Landesmus. Schloss Tirol 1 (Innsbruck 2015), 11-30. 


\section{Weiterführende Literatur}

W. Bellwald, Drei spätneolithisch/frühbronzezeitliche Pfeilbogen aus dem Gletschereis am Lötschenpass. Archäologie der Schweiz 15.4, 1992, 166-171.

J. E. Dixon/ M. Callanan/ A. Hafner/ G. P. Hare, The Emergence of Glacial Archaeology. Journal of Glacial Archaeology 1, 2014, 1-9.

J. E. Dixon/ M. F. William/ L. M. Craig, The Emerging Archaeology of Glaciers and Ice Patches. Examples from Alaska's Wrangell-St. Elias National Park and Preserve. American Antiquity, 70/1, 2005, 129-143.

M. Egg/ M. Spindler, Kleidung und Ausrüstung der Gletschermumie aus den Ötztaler Alpen. Der Mann im Eis, Band 6, Monografien des Römisch-Germanischen Zentralmuseums, Band 77 (Mainz 2009).

A. Fleckinger (Hrsg.), Ötzi 2.0. Eine Mumie zwischen Wissenschaft, Kult und Mythos (Bozen 2011).

F. Höpfel/ W. Platzer, K. Spindler (Hrsg.), Der Mann im Eis. Bericht über das Internationale Symposium 1992 in Innsbruck. Veröffentlichungen der Universität Innsbruck. Der Mann im Eis 1 (Innsbruck 1992).

A. Hafner (Hrsg.), Schnidejoch und Lötschenpass. Archäologische Forschungen in den Berner Alpen, 1/2 (Bern 2015).

A. Hafner, Archaeological Discoveries on Schnidejoch and at Other Ice Sites in the European Alps. Arctic Supplement 65/1, 2012, 189-202.

J. Junkmanns, Bogen vom Lötschenpass. In: Hafner 2015, 302-319.

L. Naef, Alpines Eis-bedrohtes Kulturarchiv. Archäologie in Graubünden 2, 2015, 161-179.

L. Naef, Eisfelder im Hochgebirge. Bedrohte Archive zur alpinen Landschaftsnutzung. In: T. Reitmaier (Hrsg.), Letzte Jäger, erste Hirten, Hochalpine Archäologie in der Silvretta, Begleitheft zur Ausstellung (Zürich 2012), 220-233.

W. Leitner/ K. Oeggl/ K. Spindler, Ein weiterer jungsteinzeitlicher Beilholm vom Hauslabjoch. Der Schlern 4/1, 2015, 4-13.

K. Oeggl/ K. Spindler, Ein weiterer neolithischer Beilholm vom Hauslabjoch. Archäologisches Korrespondenzblatt 30/1, 2000, 53-60.

S. Providoli/ P. Curdy/ P. Elsig (Hrsg.), 400 Jahre im Gletschereis. Der Theodulpass bei Zermatt und sein „Söldner“. Reihe des Geschichtsmuseums Wallis 13 (Baden 2015).

T. Reitmaier/ C. Auf der Maur/ L. Reitmaier-Naef/ M. Seifert/ C. Walser, Spätmesolithischer Bergkristallabbau auf 2800 m Höhe nahe der Fuorcla da Strem Sut (Kt. Uri / Graubünden / CH). Archäologisches Korrespondenzblatt 46/2016(2), 2016, 133-148. 
T. Reitmaier/ M. Camichel/ N. Frater/ S. Meyer/ R. Seiler/ M. Häusler/ F. J. Rühli/ M. Volken/ S. Volken, Eine weibliche Gletscherleiche aus der Zeit um 1690 aus Graubünden. Archäologie Graubünden. Archäologischer Dienst Graubünden, Amt für Kultur (Chur 2015) 13-21.

S. Schumacher, Die rätischen Inschriften. Geschichte und heutiger Stand der Forschung. Archaeolingua, Innsbrucker Beiträge zur Kulturwissenschaft, Sonderheft 79, 1992, 184.

H. Stadler, Untertan kontra Obrigkeit. Die Gletscherleiche des Wilderers Norbert Mattersberger vom Gradetzkees in Osttirol. In: J. Holzner/ E. Walde (Hrsg.) Brüche und Brücken (Wien 2005) 236-249.

H. Steiner/ C. Marzoli/ K. Oeggl, Ein Jungsteinzeitlicher Schneereif vom Gurgler Eisjoch (3134 m) im Pfossental / Schnals (Südtirol). Archäologisches Korrespondenzblatt 46/2016(4), 2016, 445-463.

H. Steiner/ L. Bezzi/ R. Gietl, Bronzezeitliche Steinbockreste zwischen Trinker- und Heuflerkogel. Gst.Nr. 5329, KG Sölden. Fundberichte aus Österrreich 51/2012, 2013, D3116-D3128.

K. Spindler/ H. Wilfing/ E. Rastbichler-Zissernig/ D. zur Nedden/ H. Nothdurfter (Hrsg.), Human Mummies. The Man in the Ice 3 (Wien, New York 1996).

M. Veith/ B. Nicolussi Castellan, Trauer fordert Gewissheit. Über den Umgang mit Gletscherleichen in Archäologie, Zeitgeschichte, Psychologie. In: H. Stadler (Hrsg.), Nearchos, Beiheft 9, Forschungen zur Gletscherarchäologie 1 (Innsbruck 2011).

J. Weisgram/ H. Splechtna/ H. Hilgers/ M. Walzl/ W. Leitner/ H. Seidler, Remarks on the anatomy of a mummified cat regarding the extent of preservation. In: K. Spindler/ H. Wilfing/ E. RastbichlerZissernig/ D. zur Nedden/ H. Nothdurfter (Hrsg.), Human Mummies. The Man in the Ice 3 (Wien, New York 1996) 289-294. 